\title{
LE VESTI E LA NUDITÀ DEL SOVRANO CHE SI CONSEGNA AL SEPOLCRO. LA MONARChIA Di Sicilia E LE STRATEGIE D'APPROCCIO ALLA CESURA DELL'EVENTO MORTE
}

\author{
Antonio Pio di Cosmo \\ Pontificio Istituto Orientale
}

Recibido: 8 de septiembre de 2018

Aceptado: 30 de septiembre de 2019

\section{Resumen}

El documento analiza el papel desempeñado por los status symbols en el funeral de los reyes de Sicilia. Esta investigación aplica el conocimiento arqueológico, antropológico y histórico, para contar la acción de la corte siciliana, que resuelve las cuestiones sobre los problemas de representación del funeral real. De esta forma, se evalúan las estrategias de comunicación orientadas al timor reventiae, que configuran las costumbres funerarias de los soberanos de Sicilia. Por lo tanto, se abre un nuevo horizonte de investigación para la fenomenología del dolor.

\section{Palabras claves}

Funeral del rey; cuerpo del soberano, status symbol, atuendo, desnudez.

\section{Riassunto}

Il contributo analizza il ruolo giocato dagli status symbols nel funerale dei re di Sicilia. Questa ricerca applica le conoscenze in materia archeologica, antropologica e storica, per raccontare l'azione della corte siciliana, che risolve le questioni circa i problemi di rappresentazione del funerale regio. In questo modo si vagliano le strategie di comunicazione orientate al timor reverentiae, che modellano i costumi dei sovrani siciliani. S'apre così ad un nuovo orizzonte di ricerca per la fenomenologia del dolore.

\section{Parole chiave}

Funerale regio, corpo del sovrano, status symbol, abbigliamento, nudità.

\footnotetext{
Pontificio Istituto Orientale, Roma. Correo electrónico: apiocosmo@outlook.it. ORCID: https://orcid. org/0000-0002-2314-4520.
} 


\begin{abstract}
This contribution analyses the role of status symbols during the burial of the kings of Sicily. This research applies archaeological, anthropological and historical knowledge. Information reported to the work of Sicilian sovereigns' entourage, which solved those issues related to the problems in representing the royal funeral. In this way, communication strategies of the timor reverentiae, which shaped the funerary customs of Sicilian sovereigns, were studied. Therefore, a new horizon in the research regarding the phenomenology of grief has opened up.
\end{abstract}

\title{
Keywords
}

Royal burial, body of sovereign, status symbol, garment, nudity.

"Ivi eran quei che fur detti felici,

pontefici, regnanti, imperadori; or sono ignudi, miseri e mendici. (...)

O ciechi, el tanto affaticar che giova?

Tutti tornate alla gran madre antica,

e 'l vostro nome a pena si ritrova".

(F. Petrarca, Trionfi, Il Trionfo della Morte, I. 81-90)

\section{Introduzione}

Il presente contributo cerca di far chiarezza sulle concrete implicazioni dell'utilizzo di un adeguato abbigliamento nei riti di inumazione dei re di Sicilia. Una narrazione che solitamente si incentra sulla formula dicotomica che contrappone il fasto delle vesti regie con cui il sovrano viene addobbato per essere consegnato al sepolcro, alla nudità del suo corpo, quale metafora d'abdicazione ed irrimediabile perdita del regno. ${ }^{2}$

Per la decodifica del tema si applica un approccio trasversale che utilizza in modo sinergico i criteri dell'archeologia, dell'antropologia e del metodo storico. Si ha a dimostrare che, oltre i silenzi e le reticenze delle fonti, la veste funebre costituisce un segno estremamente significante sia per chi partecipa al rito funerario, sia per la corte

\footnotetext{
2 Ingo Herḱotz, "Lo spazio della morte e lo spazio della sovranità", I Normanni popolo d'Europa. 1030-1200, Catalogo della Mostra, Roma, Palazzo Venezia, 28 gennaio-30 aprile 1994, M. D’Onofrio (ed.), Marsilio, Venezia, 1994, pp. 320-326; Maria Andaloro, "Per la conoscenza e la conservazione delle tombe reali della Cattedrale di Palermo: linee storiche e storicoartistiche", Il sarcofago dell'imperatore. Studi, ricerche e indagini sulla tomba di Federico II nella Cattedrale di Palermo 1994-1999, M. Andaloro (ed.), Regione Siciliana, Assessorato dei Beni Culturali, Ambientali e della Pubblica Istruzione, Palermo, 2002, pp. 135-148.
} 
che costruisce e scandisce le diverse fasi della cerimonia. Un elemento che appare indispensabile nell'adeguata composizione della salma e costituisce una 'clausola di salvaguardia' avverso il rango ostentato, in quanto è utile a reclamare la legittimità dell'esercizio dell'officio da cui l'eccellenza di status deriva. Al contempo la nudità, quale assenza dei segni ricognitori della monarchia, diviene la sua antitesi. Nel momento stesso in cui la nega, non può far altro che rivendicare con la caducità del destino umano, a cui né i re, né gli imperatori possono sottrarsi, la sua indispensabilità nell'acclarazione della sovranità rispetto alla censura dell'evento morte.

\section{La veste e la nudità: la caducità regia e le ritualità funebri}

Il tentativo di comprensione della significatività della veste rispetto al dispositivo di caducità del regnante di Sicilia diviene il punto di partenza per l'approfondimento di un tema, quale quello delle ritualità connesse al funerale del re siciliano che non ha trovato il giusto spazio nella riflessione dottrinale. Il discorso ritual-retorico sull'apparato funebre di questo sovrano medievale si instaura intorno alla dicotomia veste/nudità. Un paradosso in fin dei conti, perché lo sfarzo della veste, quale immediata esternazione della condizione regia ed imperiale dei titolari del Regno, si oppone ad un tema residuale: quello della nudità del corpo del re.

Un dispositivo che fa proprio il valore della veste, in quanto è uno strumento gnoseologico utile alla comprensione dei rapporti intercorrenti tra i membri di una precisa società. La veste difatti è il prodotto di un'“attività semiologica" e "culturale", perché assurge a "struttura strutturante" attorno a cui si organizzano le pratiche che orientano la percezione. ${ }^{3}$ Eppure l'abito è anche una "struttura strutturata" che permette di definire l'identità sociale del singolo nel segno della differenza, che introduce. ${ }^{4} \mathrm{La}$ veste esprime poi "la struttura del sistema delle condizioni" connesse all'esperienza della collocazione in una precisa posizione della struttura stessa. Quale strumento di hexis ottimizza infine la "strutturazione delle pratiche e della loro percezione".

Il valore sostanziale dell'abbigliamento esclude poi ogni ipotesi di abbigliamento alternativa, perché è un oggetto non alterabile culturalmente. E seppur è determinato storicamente dalla moda, risulta precondizionato dall'idea dell'individualità oggettiva della carica assunta. Perché quell'ordine non tollera né eccezioni, né particolarismi e nemmeno infrazioni. Siamo di fronte ad un formulario riconducibile all'idioma della regalità comune alla temperie bassomedievale, laddove la cultura si aggrega attorno al principio della trascendenza delle forme di potere.

Per questo s'arricchisce di ornamenti più preziosi, quali le insegne, il cui senso si spiega tutto nella mentalità del cosiddetto homo symbolicus. Indicatori del maggior rango e sintomi d'eccellenza, che creano "gerarchie di supremazia" e strutturano un

\footnotetext{
Pierre Bourdieu, La distinzione. Critica sociale del gusto, Il Mulino, Bologna, 1983, pp. 171; 175.

Ibidem.
}

5 Ibidem. 
sistema capace di indurre alla deferenza verso le classi dirigenti. ${ }^{6}$ La creazione di un puntuale abbigliamento difatti rientra tra quei mezzi che si oppongono ad ogni tentativo di sovversione dell'ordine costituito ed ottimizzano nel pubblico una precisa percezione della carica, acclarandone l'autorevolezza derivante dall'investitura d'origine divina. Essi appaiono utili a maggior ragione, se si considera che l'istituzione è assai giovane. Questi segni permettono poi al re locale di essere riconosciuto come tale dal gruppo dei pari sul piano nazionale ed internazionale. La veste fastosa fatta propria dal re rientra fra gli espedienti con cui si acclara la legittimità della contestata esperienza monarchica di Ruggero II, fondatore del Regno, perché costituisce con la sua materialità una 'clausola di salvaguardia' per la nuova istituzione.?

Ma questa non è certo una novità. I normanni non fanno altro che ricorrere ad una consuetudine stratificata nelle strategie di comunicazione etero-referenziale dei valori della monarchia. I politologi siciliani hanno così ad operare sull'alea semantica di segni altamente significanti, il cui senso si spalma tutto all'interno di codici ben conosciuti dall'ambito sociale. ${ }^{8}$ Tanto che si può parlare degli abiti come di 'collanti', funzionali a tenere insieme il concetto e la formula comunicativa prescelta dalla propaganda locale. Un senso tutto interno all'eclettica cultura elaborata in Sicilia, che a sua volta rimanda alla koinè costruita attorno alle soluzioni di successo di matrice bizantina.

Quest'autorevolezza deve essere ribadita dalla veste anche in quei momenti di cesura dell'esercizio della monarchia causati della dipartita del sovrano. Si può così dire che persino di fronte alla morte l'affermazione del soggetto si attua attraverso l'abbigliamento

6 Rocco Antonio CARILE, "La sacralità rituale dei BA $\Sigma \mathrm{I} \Lambda \mathrm{EI} \Sigma$ bizantini”, Per me reges regnant. La regalità sacra nell'Europa medievale, F. Cardini y M. Saltarelli (edd.), il Cerchio-Cantagalli, Rimini-Siena, 2002, p. 53.

7 Fulvio Delle Donne, "Liturgie del potere. Le testimonianze letterarie", Nascita di un regno. Poteri signorili, istituzioni feudali e strutture sociali nel Mezzogiorno normanno (1130-1194), Atti delle diciassettesime Giornate normanno-sveve, Bari, 10-13 ottobre 2006, R. Licinio y F. Violante (edd.), Dedalo, Bari, 2008, pp. 331-368; Wiliam Tronzo, The Cultures of His Kingdom. Roger II and the Cappella Palatina in Palermo, Princeton University Press, Princeton, 1997; Ernst Kantorowicz, "On the Portrait of Roger II in the Martorana in Palermo”, Proporzioni. Studi di storia dell'arte, III, Sansoni, Firenze, 1950, pp. 30-35; Ernst Kantorowicz, I mosaici di Monreale, Flaccovio, Palermo, 1960; Ernst Kantorowicz, "Some Reflections on Portraiture in Byzantine Art", The Art of Byzantium and the Medieval West: Selected Studies, W.E. Kleinbauer (ed.), Indiana University Press, Bloomington-London, 1976, pp. 256-269; Ernst Kantorowicz, "The Byzantine Contribution to Western Art of the Twelfth and Thirteenth Centuries", The Art of Byzantium and the Medieval West: Selected Studies, W.E. Kleinbauer (ed.), Indiana University Press, Bloomington-London, 1976, pp. 357-388; Ernst Kantorowicz, I mosaici di Santa Maria dell'Ammiraglio a Palermo, Istituto Siciliano di Studi Bizantini e Neoellenici, Palermo, 1990; Ernst Kantorowicz, "La Cappella Palatina di Palermo. I mosaici del Presbiterio", I mosaici del periodo normanno in Sicilia, E. Kitzinger (ed.), Istituto Siciliano di Studi Bizantini e Neoellenici, Palermo, 2000a; Ernst Kantorowicz, "La cattedrale di Cefalù. La cattedrale di Palermo e il Museo Diocesano. Mosaici profani", I mosaici del periodo normanno in Sicilia, VI, E. Kitzinger (ed.), Istituto Siciliano di Studi Bizantini e Neoellenici, Palermo, 2000b.

8 René KönIG, Il potere della moda, Liguori, Napoli, 1992; Jane SchneIder, The Antropology of Cloth, Annual Review of Anthropology, 16 (1987), pp. 409-448. 
indossato, concretizzandosi nella misura in cui le forme culturali lo permettono. S'apre ad un'assolutizzazione del valore dell'abito, quale peculiare status symbols che esplica la sua azione anche nell'ambito dei riti funerari.

La riflessione sull'abbagliamento funebre dei re di Sicilia, ed in particolare di Enrico VI e Federico II imperatori, implica poi la rivisitazione delle strategie di rappresentazione della regalità locale e al contempo della sovranità universale del Medioevo occidentale; una riflessione che investe ancora le relative strutture dell'universo mentale dell'uomo medievale tout court, che concepisce l'abbigliamento come mezzo principale per la ricognizione del rango.

Un discorso sulla caducità che costituisce a sua volta un tentativo di razionalizzazione del mondo e conseguente sistematizzazione, entro uno schema fatto proprio da un preciso sistema rappresentativo come quello dello Stato bassomedievale, che si raffronta con l'evento morte.

Il dispositivo della caducità ricorre allora all'alea semantica della veste e al suo valore indicale, utile ad esternare il rango, in quanto costituisce un formalismo dal valore sostanziale. Esso diviene imprescindibile in un'epoca come il Medioevo, in cui la forma appare indispensabile per rendere percepibile la sostanza. Formalismi che appaiono fondamentali quando i sovrani sono vivi, ed ancor più dopo il loro decesso.

Deve poi considerarsi una realtà come quella della Sicilia normanna, laddove i regnanti sono attentissimi agli status symbols, specie a quelli fatti propri dalle diverse tradizioni concernenti la monarchica assoluta. Si osserva come tutti questi vengono posti alla base dell'esperienza del giovane regno siciliano e giustificano l'esercizio della monarchia; a maggior ragione allorché la morte ne nega la continuità.

La necessità di somministrazione di precise idee genera un'imprescindibile esigenza: calibrare attentamente qualità e quantità dei decori della veste da opporre all'evento morte, inserendo il dettaglio prezioso in un contesto sociologicamente e culturalmente determinato come il funerale. L'ostentazione al pubblico degli attributi di potere assume allora maggior senso nei tempi dell'atto rituale della prothesis; una comportamentalità che anche in Sicilia la dottrina del potere tende a sacralizzare attraverso l'utilizzo di un protocollo preciso e ben scandito. Sicché il rito funebre, da intendersi alla stregua di uno spettacolo e di una rappresentazione drammatizzata come tutti gli altri momenti 'forti' dell'eventologia, può giocare sulle fogge dell'abbigliamento, sulla significatività della cromia e sulla ricchezza dei materiali, quali potenti evidenziatori visuali.

La morte, orbene, carica questi segni di un ulteriore valore ontologico portandolo al parossismo, quale concreta espressione di quell'ipertrofia del simbolo che connota i riti funerari, allo scopo di neutralizzare col suo senso gli effetti del decesso.

L'operazione di decodifica dei codici culturali di cui l'abito si fa vettore in questo specifico momento della vita dello Stato vuole infine penetrare i tre sub-aspetti su cui l'alea di questo segno riposa: il didattico-esplicativo, il mistico-sacrale, il sociale-psitico. Si apre pure ad una sociologia delle rappresentazioni dei simboli del potere, fra cui rientra la veste. Segni che soddisfano il bisogno di distinzione e affermazione di un soggetto che 
si auto-rappresenta rispetto alla classe di appartenenza: l'aristocrazia e avverso l'ordine a cui pertiene: la "familia regum". ${ }^{9}$ Esigenza che col decesso si fa più pregnante. La veste ha a ribadire rispetto alla morte la legittimità del rango vantato, quale condizione di esistenza dell'officio che il re siciliano svolge anche a dispetto dell'intero ordo regum. Una posizione contestata, fino a che il regno locale non viene assorbito col trattato di Venezia nel Sacro Romano Impero e la sua corona assunta dall'imperatore occidentale.

\section{La veste e l'evento morte: cautele metodologiche e problematiche d'approccio}

La comprensione del valore sociologico della veste e del suo opposto, la nudità, in un momento fondamentale della vita del regno come il lutto di Stato pone alcune problematiche d'approccio.

Innanzitutto manca una solida linea di ricerca che ha investigato in maniera approfondita il tema. Pertanto, in assenza di elementi adeguati con cui poter puntellare le ipotesi, si è costretti ad effettuare un complesso lavoro di collazione delle fonti, volto ad isolare un preciso aspetto, quello legato all'abbigliamento, che spesso si nasconde fra le pieghe di un più complesso racconto. ${ }^{10}$ Un lavoro d'esegesi dunque, che si confronta con i silenzi, le reticenze o comunque con il non detto. Silenzi che emergono prepotenti durante la lettura dei racconti delle dipartite dei sovrani della dinastia degli Altavilla. Diversamente per gli Svevi si nota una maggiore attenzione, non solo per il vivido

\footnotetext{
Franz DöLGER, “Die Familie der Könige im Mittelalter”, Historisches Jahrbuch 60 (1940), pp. 397-420; Franz DöLger, "Brüderlichkeit der Fürsten”, Reallexikon für Antike und Christentum, vol. 2, Hiersemann, Stuttgart, 1954, col. 642; Wolfram Brandes, "Die 'Familie der Könige' in Mittelalter”, Diskussionsbeitrag zur Kritik eines vermeintlichen Erkenntnismodells, Rechtsgeschichte/Legal History, 21 (2013), pp. 262-284. 10 Léon-Robert MÉNAGER, "L'institution monarchique dans les États normands d'Italie. Contribution à l'étude du pouvoir royal dans les principautés occidentales, aux XI-XII siècles", Hommes et institutions de l'Italie normande, Léon-Robert Ménager (ed.), London, 1981, pp. 303-331; 445-468; Dieter MerTens, Il pensiero politico medievale, Il Mulino, Bologna, 1999; Paolo Delogu, "Idee sulla regalità: l'eredità normanna", Potere, società e popolo tra età normanna ed età sveva (1189-1210), Atti delle Quinte Giornate Normanno-Sveve, Bari-Conversano, 26-28 ottobre 1981, Dedalo, Bari, 1983, pp. 185-214; Paolo Cammarosano, "Immagine visiva e propaganda nel Medioevo", I linguaggi della propaganda. Studio di casi: Medioevo, Rivoluzione Inglese, Italia liberale, Fascismo, Resistenza, Mondadori, Milano, 1991, pp. 8-29; Glauco Maria Cantarella, "Historia non facit saltus? Gli imprevisti normanni", I re nudi. Congiure, assassini, tracolli ed altri imprevisti nella storia del potere, G. M. Cantarella y F. Santi (edd.), Centro Italiano di Studi sull'Alto Medioevo, Spoleto, 1996, pp. 9-38; Glauco Maria Cantarella, La Sicilia e i Normanni. Le fonti del mito, Patron, Bologna, 1988; Glauco Maria Cantarella, Principi e corti. L'Europa del XII secolo, Einaudi, Torino, 1997; Glauco Maria CAnTarella, "Il pallottoliere della regalità: il perfetto re della Sicilia normanna", Studi in onore di Vincenzo D'Alessandro, Dentro e fuori la Sicilia, P. Corrao y I. E. Mineo (edd.), Viella, Roma, 2009, pp. 29-44; Glauco Maria Cantarella, Medioevo. Un filo di parole, Mondadori, Milano, 2002; Glauco Maria CAntarella, "Le basi concettuali del potere", Per me reges regnant. La regalità sacra nell'Europa medievale, F. Cardini y M. Saltarelli (edd.), il Cerchio-Cantagalli, Rimini-Siena, 2002, pp. 193-207; Glauco Maria Cantarella, "Divagazioni preliminari”, "C'era una volta un re...» Aspetti e momenti della regalità, Seminario del Dottorato in Storia Medievale dell'Università di Bologna, Bologna, 17-18 dicembre 2003, G. Isabella (ed.), Clueb, Bologna, 2005, pp. 9-24.
} 
ricordo che il corteo funebre lascia negli avventori come Spinelli, ma perché la morte di Federico II in particolare richiede cautele eccezionali, data la scomunica. Altrettanto incisivo deve poi apparire il discorso sulla caducità del potere che si incarna nella nudità di Manfredi, che sconfitto a Benevento è privato di tutto, non solo del fastoso tesoro che finisce nelle mani degli Angiò, ma persino degli indumenti che lo rivestono. Un tema retorico, introdotto da un cronista filo-ecclesiastico come Villani che vuol dire molto di più, perché propone un'efficace metafora della transitorietà del potere, inserita in un più generale clima, che già dalla metà del sec. XI, genera un preciso apparato di caducità per i pontefici. ${ }^{11}$

Un memento mori forse non troppo alieno alla coscienza comune, specie in un mondo in cui l'età media della morte non è troppo avanzata. Una fattualità che statisticamente è confermata dalla brevità di vita dei sovrani di Casa Altavilla e pure da quella degli Svevi. Una serie di idee che presto sembrano attecchire anche negli apparati della regalità laica, fin tanto che la veste e la nudità costituiscono dei codici fatti propri dall'immaginario del tempo del lutto. Soluzioni insomma, utili a fomentare la propaganda ed il suo contrario, perché si muovono su più livelli di significato.

La dialettica veste/nudità si riscontra finanche negli ampli riferimenti agli atti penitenziali che precedono il decesso o persino nelle narrazioni affabulatorie, che culminano nella formositas di Guglielmo II, che seppur morto sembra essere vivo, mentre conserva una bellezza straordinaria. Un 'fiore della retorica' che traduce una rappresentazione ideale della monarchia, perché trasforma la salma regia a seguito dell'evento morte in una concreta espressione del corpo politico del re; in tal modo si crede di neutralizzare gli effetti della fine del ciclo biologico a cui nemmeno i re possono sottrarsi. Guglielmo II allora ha ad incorporare nella sua fisionomia un'idea, quella della maestà locale, che pretende di essere autocratica. Un espediente che si oppone all'apparato di caducità, lo trascende e lo incorpora, alludendo all'eternità della monarchia. Una pretesa che emerge tra le pieghe dei versi di Pietro da Eboli e sembra soddisfarsi solo una volta che il Regno è sussunto entro l'Impero.

Occorre stigmatizzare un ulteriore aspetto che si muove a corollario di alcuni di questi silenzi e, al contempo, si oppone ad essi. L'evento morte, stando a quanto dimostrato dagli indicatori archeologici e letterari, obbliga a manifestare l'abbondanza regia, quale esigenza antropologicamente connotata; costituisce poi un segno concreto della felicitas del sovrano. Un'abbondanza che coinvolge col suo sentore uniformante anche i riti funesti e trova dei segnacoli primari nelle insegne del potere opportunamente ostentate, che operano come una 'clausola di salvaguardia'. ${ }^{22}$ Essi implicano non solo il legittimo

\footnotetext{
11 Agostino Paravicini Bagliani, Le Chiavi e la Tiara. Immagini e simboli del papato medievale, Viella, Roma, 1998, pp. 85-93.

12 Percy Ernst Schramm, "Herrschaftszeichen und Staatssymbolik. Beiträge zu ihrer Geschichte von dritten bis zum sechzehnten Jh., I-III", Early Medieval Europe, Vol. 3, Stuttgart, 1956, pp. 135-156; Reinhard ElzE, "Insegne del potere sovrano e delegato in Occidente", Simboli e simbologia nell'Alto Medioevo, Atti della XXIII Settimana di Studio del Centro Italiano di Studi sull'Alto Medioevo, Spoleto, 3-9 aprile 1975, Centro Italiano di Studi sull'Alto Medioevo, Spoleto, 1976, pp. 569-593; Reinhard Elze, "Le insegne del potere",
} 
esercizio della monarchia, ma costituiscono al contempo il pegno d'eternità del corpo politico regio, a tutta garanzia della continuità dell'istituzione. Una necessità sentita a maggior ragione, perché in Sicilia questo momento delicato per la vita dello Stato viene segnato solitamente dal tumulto.

L'ostentazione di abbondanza esige poi la devoluzione di risorse all'effimero, che devono caratterizzare tanto le epifanie pubbliche del sovrano, quanto le cerimonie che vengono poste in essere per opporsi ai momenti di crisi. L'ostentazione rituale nutre le strategie di auto-rappresentazione degli episodi della regalità ed ottimizza una prassi politica volta ad ottenere il consenso. Sicché le manifestazioni di grandeur appaiono come un 'farmaco' da utilizzare nei momenti nodali della vita del Regno e specie in quelli di rottura. Queste cerimonie, fra cui rientra il funerale fastoso, configurano degli atti tutti politici, che servono ad 'oliare' opportunamente il meccanismo del consenso. Essi appaiono per lo più necessari, perché richiesti da quelle formule che strutturano la percezione del potere per quel che è nei diversi strati sociali. ${ }^{13}$

Le strategie di spettacolarizzazione implicano più di una cautela nella progettazione $\mathrm{e}$ realizzazione della messa in scena. Difatti il dispendio rituale non deve urtare la sensibilità dello spettatore, specie di quella gente comune che deve essere maggiormente convinta dalla cerimonia. La devoluzione all'effimero delle risorse è finalizzata ad orientare la coscienza dell'uomo comune, porla sotto controllo, anche a mezzo della meraviglia opportunamente suscitata, come se ivi vi fosse qualcosa di magico. Un meccanismo che utilizza persino il timore come strumento di consenso.

Ma questa è solo una faccia della medaglia. Occorre far propria un'ulteriore cautela. Bisogna anche guardare alla narrazione dell'evento stesso, perché è frutto di una o più mediazioni. L'impressione generata nell'animo del diretto osservatore è ben differente da quella prodotta in coloro che, a loro volta, lo recepiscono da altrui, per trasmetterlo poi con lo scritto. Questi operano su una materia fallace come la memoria, selezionando episodi, dettagli e, quando occorre, ampliando o aggiungendo dei particolari che vanno a farsi sempre più incisivi; fin tanto da scadere in una vera e propria mancanza di dati oggettivi. L'estrapolazione dei dati si limita allora alla possibilità di apprezzare una serie di informazioni che hanno colpito i narratori. Siamo di fronte ad una duttilità

Strumenti, tempi e luoghi di comunicazione nel Mezzogiorno normanno-svevo, Atti delle Undicesime Giornate Normanno-Sveve, Bari, 26-29 ottobre 1993, Dedalo, Bari, 1995, pp. 113-129; Salvatore Tramontana, Vestirsi e travestirsi in Sicilia. Abbigliamento, feste e spettacoli nel Medioevo, Sellerio, Palermo, 1993, pp. 86-98; Rotraud BAuER, "Il manto di Ruggero II", I Normanni popolo d'Europa. 1030-1200, Catalogo della Mostra, Roma, Palazzo Venezia, 28 gennaio-30 aprile 1994, M. D’Onofrio (ed.), Marsilio, Venezia, 1994, pp. 278-287; Rotraud BAUER, "Il manto di Ruggero II e le vesti regie", Nobiles Officinae: perle, filigrane e trame di seta dal Palazzo Reale di Palermo, M. Andaloro (ed.), Maimone, Catania, 2006a, pp. 171-180; Rotraud BAUER, "Le vesti e le insegne per l'incoronazione dei re e degli imperatori del Sacro Romano Impero", Nobiles Officinae: perle, filigrane e trame di seta dal Palazzo Reale di Palermo, M. Andaloro (ed.), Maimone, Catania, 2006b, pp. 425-429; Mirko Vagnoni, Dei gratia rex Sicilie. Scene d'incoronazione divina nell'iconografia regia normanna, Federico II University Press, Napoli, 2017; Ernst Kantorowicz, "On the Portrait...", pp. 30-35.

13 Salvatore Tramontana, L'effimero nella Sicilia normanna, Sallerio, Palermo, 1984, pp. 17-23. 
della costruzione del racconto, che implica anche una serie di trucchi nella strutturazione della memoria, per il cui tramite si rivisitano in modo più o meno volontario gli eventi. Racconti che in ultima analisi costituiscono un punto di vista, quello del committente, il quale orienta le finalità narrative. ${ }^{14}$

Tutta l'aneddotica concernente il tempo del lutto difatti si afferisce alla produzione dei ceti dominanti, costituendo un discorso endo-referenziale, quale sottoprodotto della dialettica politica nazionale prima ed internazionale poi. Storie che vanno ricondotte alle esigenze della nobiltas e del clero, perché affidate ai suoi intellettuali, che sono capaci di maneggiare quello strumento, mentre si fanno inevitabilmente portatori di istanze culturali e comportamentali di 'classe'.

La ricerca soffre un ulteriore limite nella ricostruzione dell'effettivo corredo funebre dei sovrani siciliani: la difficoltà di conservazione di un dato della cultura prettamente materiale, quale il tessuto. Le stoffe per loro natura vivono una breve vita e appaiono particolarmente fragili nel lungo periodo. Sicché il contributo che l'archeologia può offrire risulta modesto e persino deludente. ${ }^{15}$ Le evidenze sopravvissute poi difficilmente sono apprezzabili nello splendore originario, ma piuttosto offrono quel colore impostogli dal tempo. Ma vi è di più. Per quel che riguarda il corredo funebre un ruolo fondamentale è giocato dai processi di conservazione del corpo e dalle sostanze usate a tale scopo, che contribuiscono all'alterazione della condizione originaria dei tessuti.

Appare così utile alla ricostruzione della foggia della veste funebre una fonte postuma: "I regali sepolcri del duomo di Palermo riconosciuti ed illustrati", edito nel $1784 \mathrm{e}$ curato da Francesco Daniele, che descrive le vesti di Ruggero II e dei sovrani Svevi tumulati nella cattedrale di Palermo. Testo che riempie i vuoti lasciati dal silenzio delle fonti più o meno coeve ai fatti e fornisce informazioni indispensabili per comprendere le consuetudini pertinenti l'abbigliamento adoperato in morte dai sovrani. Tale fonte purtroppo si limita alla ricognizione dei capi ritrovati nel sepolcro ed omette ogni riferimento alla mise adoperata durante la prothesis rituale e, d'altronde, non può farlo. La fonte soffre di un altro limite: l'autore non è avvezzo al gusto medievale e le sue descrizioni tendono nonostante le anatomiche descrizioni, espressione dell'epoca dei Lumi che ha prodotto l'opera, a non porre la giusta attenzione alla significatività ideologica della manifattura.

Altrettanto utile appare un'altra fonte edita nel 1821: "I discorsi intorno alla Sicilia" di Rosario Gregorio, che fa opportuna menzione degli indumenti utilizzati nell'inumazione dei sovrani sepolti a Palermo. Anche questi pecca di una scarsa conoscenza del gusto medievale e pertanto non mette in giusta luce i dettagli preziosi delle evidenze tessili.

Fulvio Delle Donne, "Liturgie del potere...", p. 336.

15 Salvatore Tramontana, Vestirsi e travestirsi..., p. 48; Giancarlo Andenna, "Dalla legittimazione alla sacralizzazione della conquista (1042-1140)", I caratteri originari della conquista normanna. Diversità e identità nel Mezzogiorno (1030-1130), Atti delle Sedicesime Giornate Normanno-Sveve, Bari, 5-8 ottobre 2004, Dedalo, Bari, 2006, pp. 371-405; Paolo Delogu, "Idee sulla regalità..., pp. 185-214. 
Entrambe le opere mettono di fronte ad un punto di vista viziato. La veste della tumulazione solitamente non richiede lo stesso dispendio di risorse adoperato per la prothesis del sovrano, ma appare ben più dismessa. Ciò non permette di percepire in modo adeguato quel senso di spettacolarizzazione fatto proprio da queste cerimonie del tempo del lutto. Balzano all'attenzione atti quali la sostituzione della corona e delle altre insegne con elementi in materiali più poveri, come l'argento dorato. ${ }^{16}$ Per la deposizione della salma difatti si pone in essere una strategia volta al risparmio di risorse, che altrimenti andrebbero inutilmente perse in quello che è nulla più di un sacrificio rituale a favore del defunto. Una realtà che la stessa salma di Federico dimostra: i vestimenta regalia in quest'occasione vengono sostituiti da vestimenta leviora, un corredo di corte che non lascia certo venire meno quello sfarzo tipico della condizione imperiale, ma si orienta alla salvaguardia di beni, la cui inutile devoluzione viene scongiurata avverso le ritualità d'espulsione dalla compagine sociale.

\section{La sepoltura di Ruggero II e l'ergasterion palermitano: i motivi decorativi della tradizione bizantina e la veste funebre del re}

Il resoconto concernente lo stato della sepoltura di Ruggero II, opera di Rosario Gregorio, appare di particolare interesse, specie se si confrontano le brevi informazioni evinte dal testo con quanto racconta Alessandro di Telese per il rito di incoronazione del medesimo sovrano. Il telesino ha difatti cura di descrivere nei particolari la preziosità delle stoffe $\mathrm{e}$ il dispendio altrettanto rituale delle risorse consumate in quell'occasione. ${ }^{17}$ Un'attenzione al dettaglio che nello schematico resoconto di Gregorio sembra mancare. Eppure il fine ideologico di Alessandro è diverso: questi è impegnato a delineare un'apologia e la profusione dei beni trova un suo equilibrio entro un complesso costrutto retorico, che costituisce piuttosto un esercizio dialettico volto a perorare la legittimità dell'istituzione. Un'assenza nella deposizione che però mette in dubbio il perseguirsi di una determinata politica e di un 'marchio' dell'azione di Ruggero II, quale il dispendio di risorse volte all'effimero, decretandone l'insuccesso. ${ }^{18}$ Un fallimento in politica economica dunque, che sembra rispecchiarsi nella sua deposizione. Ma è davvero così?

\footnotetext{
16 Angelo Lipinsky, "Le insegne regali dei sovrani di Sicilia e la scuola orafa palermitana", Atti del Congresso Internazionale di Studi sulla Sicilia Normanna, Palermo, 4-8 dicembre 1972, Caltanissetta-Roma, Istituto di Storia Medievale Università di Palermo, Sciascia, 1973, pp. 162-194; Angelo Lipinsky, Oro, argento, gemme e smalti. Tecnologia delle arti dalle origini alla fine del Medioevo. 3000 a. C. - 1550 d. C., Olschki, Firenze, 1975.

17 Alexander Telesinus, De rebus gestis Rogerii Siciliae regis (1127-1135), III, 4; 1; Salvatore Tramontana, L'effimero nella Sicilia..., p. 26.

18 Paolo Delogu, "La committenza degli Altavilla: produzione monumentale e propaganda politica", The Mosaics of Norman Sicily, O. Demus (ed.), Hacker Art Books, New York, 1988; Salvatore Tramontana, L'effimero nella Sicilia ... , pp. 18-23; Salvatore Tramontana, Vestirsi e travestirsi... , pp. 138-148; Salvatore Tramontana, "Comunicare nel Mezzogiorno", Strumenti, tempi e luoghi di comunicazione nel Mezzogiorno normanno-svevo, Atti delle Undicesime Giornate Normanno-Sveve, Bari, 26-29 ottobre 1993, Dedalo, Bari, 1995, pp. 9-30; Hubert Houben, Ruggero II di Sicilia. Un sovrano tra Oriente e Occidente, La Terza, Roma- Bari, 1999.
} 
Deve considerarsi che i dati rilevabili da questo resoconto soffrono di un gap culturale. Sembra emergere un preciso atteggiamento intellettuale, riconducibile all'humus culturale del periodo che produce questo resoconto. Ciò non permette di percepire lo sforzo profuso nell'approntare una strategia di grandeur volta ad accompagnare il re nella morte. Un fasto che non riesce a trasparire anche perché il redattore del testo non è affatto educato al gusto medievale, alla peculiarità dei suoi elaborati tessili, nonché alla simbologia rappresentata. Tale programma iconografico che connota i tessuti viene liquidato semplicisticamente come stranezza e descritto con quella diffidenza "etnocentrica", con cui si è soliti approcciare i reperti che si mostrano ad un occhio non abituato a quel preciso gusto. L'evidenza assume un carattere esotico, che non gli rende giustizia.

La descrizione appare così vaga e poco puntuale, specie nell'approccio al dato visuale:

"trovaronsi nel sepolcro lunghissimi stracci di velo giallo: molti erano annodati tra loro, ed altri di essi avevano all'estremità dei fregetti di oro intessuto. Vi era anche uno straccio di drappo, una parte del quale è di colore giallognolo, l'altra è fregiata, dipinta, e screziata di bei colori a veder vaghi: l'artifizio ne è pur sottilissimo, ma è rozzo e strano il disegno, e rappresenta varj animali, uomini a cavallo e altre figure" ${ }^{19}$

Il frammento di tessuto inquadra entro un motivo serpentiforme una serie di fiere sia reali, sia fantastiche che, insieme a due cavalieri, si dispongono dentro gli spazi disegnati dal corpo squamato del mostro che trattiene tutti i partecipanti alla caccia. Il complesso disegno si inserisce entro due bande decorate a motivo zoomorfo.

Nonostante ciò si riesce comunque a rilevare la qualità della fattura del tessuto, che perpetua i motivi decorativi tipici delle sete bizantine ed orientali in generale. Sete che sicuramente vengono prodotte nell'ergasterion palermitano, laddove operano maestranze ascrivibili al melting pot che anima la Sicilia del periodo. Si ripropongono nel locale gli out put bizantini, che fanno dei tessuti dei veri e propri 'sottoprodotti' della regalità di Bisanzio, utili alle strategie di auto-rappresentazione del sovrano siciliano..$^{20}$

19 Gregorio Rosario, Discorsi intorno la Sicilia, Pedone, Palermo, 1923, p. 25.

20 Mirko VAGNONI, "Problemi di legittimazione regia: "imitatio Byzantii", Il papato e i Normanni: temporale e spirituale, convegno di studi, Ariano Irpino 6-7 dicembre 2007, E. D’Angelo y C. Leonardi (edd.), SISMEL edizioni del Galluzzo, Firenze, 2011, pp. 50-65; Georgess Didi-Huberman, "Imitation, représentation, fonction. Remarques sur un mythe épistémologique", L'image. Fonctions et usage des images dans l'Occident médiéval, Actes du 6e International Workshop on Medieval Societes, Erice, 17-23 octobre 1992, J. Baschet y J.C. Schmitt (edd.), Le Léopard d'Or, Paris, 1996, pp. 59-86; Agostino Pertusi, "Insegne del potere sovrano e delegato a Bisanzio e nei paesi di influenza bizantina", Simboli e simbologia nell'Alto Medioevo, Atti della XXIII Settimana di Studio del Centro Italiano di Studi sull'Alto Medioevo, Spoleto, 3-9 aprile 1975, Centro Italiano di Studi sull'Alto Medioevo, Spoleto, 1976, pp. 481-568; André Grabar, L'empereur dans l'art byzantin. Recherches sur l'art officiel de l'Empire d'Orient, Les Belles Lettres, Paris, 1936; Adele Cilento y Filippo Burgarella, Bisanzio in Sicilia e nel

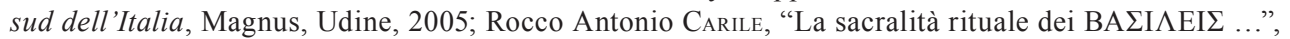


Si osserva allora come la regalità di Ruggero non può fare a meno dei prodotti culturali di Bisanzio e dei suoi motivi, giacché le scene di caccia ed i diversi animali a cui si allude, appartengono a quell'immaginario consolidato della regalità e condiviso dall'uomo medievale. Un'imaginerie consueta a cui si ricorre per ribadire nel momento di cesura dovuto alla morte la legittimità della propria esperienza regale, vessata non solo in fatto, ma soprattutto in diritto. L'evocazione di un repertorio tradizionale appare dunque come motivo apologetico. Sul piano sociologico, al contempo, il tessuto ricercato con i suoi materiali preziosi ha a confermare lo status, che nonostante la morte non viene definitivamente perso. La cura nei materiali e l'utilizzo di dettagli preziosi costituisce una 'clausola di salvaguardia'. La texture dell'abbigliamento, tanto quanto il motivo decorativo, diviene un pegno di legittimità, perché si oppone con la sua preziosa materialità alle pretese avverse dei due imperatori e del papa, come fa sul piano concettuale l'antico tema della caccia, già fatto proprio dal repertorio affabulatorio della regalità.

L'evidenza tessile riconducibile alla veste regale di re Ruggero, oggi perduta nella sua interezza, va necessariamente ascritta fra i più antichi documenti dell'ergasterion. La sua esecuzione difatti ha come termine ante quem il 1154 d.C., anno della morte. Il motivo decorativo e la qualità di trama ed ordito permettono di instaurare una serie di processi comparativi con gli altri reperti tessili più o meno coevi, che ad esso possono assimilarsi in ragione della loro lavorazione. ${ }^{21}$

I tessuti rinvenuti nella tomba fungono poi da strumento probatorio, che dimostra la diffusione dei 'motivi erranti della regalità' elaborati dalla cultura materiale bizantina. Ciò a maggior ragione, se si postula pure l'intervento di presunte maestranze bizantine nella confezione di queste stoffe o, comunque, di ricamatori arabi avvezzi ad un gusto diffuso nel Mediterraneo.

Si raffronta così una più incisiva colonizzazione dell'immaginario locale da parte delle elaborazioni bizantine, che si rispecchia nella cultura materiale in generale e nella produzione serica in particolare. Ciò è dovuto all'apprendimento di tecniche di lavorazione, che si realizza in modo più incisivo nel cosiddetto "secondo periodo" dell'ergasterion palermitano, allorché l'opificio regio può contare su quelle maestranze muliebri greche o bizantinofone, condotte a Palermo dopo le azioni belliche nel Peloponneso.

\footnotetext{
pp. 53-95; Rosalia Varoli Piazza, "La produzione di manufatti tessili nel Palazzo Reale di Palermo: 'tiraz' o 'ergasterion'”, I Normanni popolo d'Europa. 1030-1200, Catalogo della Mostra, Roma, Palazzo Venezia, 28 gennaio-30 aprile 1994, M. D’Onofrio (ed.), Marsilio, Venezia, 1995, pp. 288-290.

21 Rosalia Varoli Piazza, "La produzione di manufatti tessili..., p. 290; Rosalia Varoli Piazza, "Per un approccio interdisciplinare ai manufatti tessili antichi”, Nobiles Officinae: perle, filigrane e trame di seta dal Palazzo Reale di Palermo, M. Adaloro (ed.), Maiomone, Catania, 2006, pp. 393-395.
} 
FIG. 1.

\section{Frammento del tessuto della veste di Ruggero II, xilografia da F. Daniele, I reali sepolcri di Palermo riconosciuti ed illustrati, Palermo, 1784, Tav. C.}

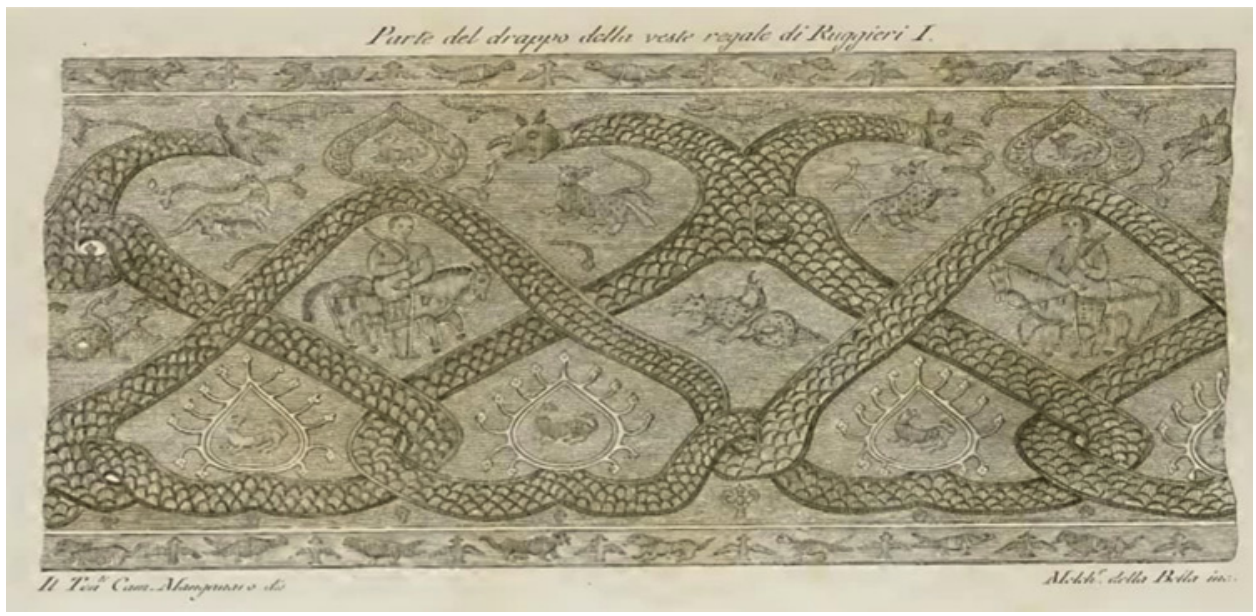

\section{I riti funebri per Guglielmo I: la cerimonia e le esigenze della politica}

Altrettanta vaghezza e penuria di dettagli si osserva nella narrazione del vescovo salernitano, Romualdo II Guarna, il quale si preoccupa di descrivere nella sua cronaca non il funerale, ma gli atti di penitenza e le disposizioni testamentarie di Guglielmo I morente. Romualdo II riferisce informazioni sicuramente utili per lo storico e l'antropologo, allorché racconta i comportamenti ritualizzati nell'attendere la morte, ma nulla dice dell'abbigliamento funebre, né lascia intuire un qualche indizio utile alla ricostruzione dello stesso. Per questo poco o nulla si può dire, se non genericamente riferire del rispetto di una consuetudine leggittimatrice e dell'ossequio di una prassi volta a tutelare la particolare dignità del defunto. Questa ipotesi appare verosimile nonostante la sobrietà e la cauta politica tendente ad accumulare risorse, che è cifra peculiare del regno di Guglielmo I.

Più saporosi dettagli arrivano invece da Falcando, che narra gli espedienti adoperati dalla corte al momento della morte di Guglielmo I. La regina e l'entourage temono atti di rivolta da parte di una plebe vessata, che facciano precipitare il regno nel caos. Pertanto costoro, seguendo un'antica consuetudine che accompagna la dipartita dei re, scelgono di nascondere la morte del sovrano, diffondendo la notizia solo con effettivo ritardo:

"...decisero di far apparire che il re fosse ancora in vita e di nascondere provvisoriamente il feretro nello stesso palazzo fino a tanto che l'arrivo dei 
baroni, e quindi la partecipazione corale della nobiltà, non fosse pronto tutto quel che era necessario per l'incoronazione del successore. "22

Se ne deduce che l'organizzazione dei funerali deve rientrare fra quegli atti volti a costruire il consenso. Apprensioni giustificate dalla travagliata esperienza della monarchia locale, che si fa particolarmente dolorosa sotto Guglielmo I. È interesse della stessa corte fornire un'immagine dismessa della monarchia, attraverso l'insistenza sul pubblico cordoglio da manifestare nelle modalità rituali divenute consuete nei funerali. Una messa in scena dunque, che vuole restituire una realtà ideale.

E se il popolo attraverso questi atti può essere opportunatamente "orientato", ${ }^{23}$ ben diversa è la realtà vissuta dai membri della curia regia e dei vescovi, i cui sentimenti mesti, per lo più coartati, poco hanno a che fare con la pietà verso il defunto. ${ }^{24}$ Queste espressioni del dolore si riconducono piuttosto agli espedienti della 'macchina del consenso', che l'istituzione è costretta ad attivare di fronte all'evento morte. $\mathrm{O}$ almeno così Falcando vuol far credere che sia andata.

La ricostruzione del rito approntato per Guglielmo I diventa più ostica, perché si confronta con i silenzi, le reticenze e persino con la mistificazione delle cronache, che fin troppo malevolmente interpretano le vicende concernenti questo sovrano. Una presunzione che si fa particolarmente verosimile, allorché si prende in considerazione l'incisiva kaiserkritik posta in essere da uno scrittore come Falcando. Un leitmotiv eccessivamente gradito al suo committente, che appare estremamente ostile alla Casata degli Altavilla e alle loro azioni politiche. Tant'è che impegna l'autore nell'evidenziare le sole attività della corte volte a raccattare consensi avverso un sovrano, che egli presenta come molto impopolare. Queste velleità narrative fanno passare sotto silenzio tutti gli atti rituali del funerale, che vengono liquidati rapidamente, perché sembrano interessare poco o nulla Falcando:

"Quod ubi paucis diebus peractum est, primum in palatio more lugubri funere conclamato repente totius luctus subsequutus est civitatis (...), de loco ubi sepultum fuerat, in capellam corpus regium transtulerunt". ${ }^{25}$

Eppure la 'macchina del consenso' non dimentica gli elementi rituali del tempo del lutto: largo spazio viene dato nel racconto di Falcando alla fenomenologia del dolore propria del rito, che viene affidata alle donne e nello specifico a quelle saracene. Le quali per tre giorni mostrano cordoglio:

\footnotetext{
Falcandus, Liber de Regno Siciliae, 88, XXV.

Salvatore Tramontana, L'effimero nella Sicilia ... , pp. 32-33.

Ivi, 33-34.

Falcandus, Liber de Regno Siciliae, 89, XXV.
} 
"Universi quoque cives nigris induti vestibus usque in diem tertium in eodem habitu permanserunt. Per tolum autem hoc triduum mulieres, nobilesque malronae, maxime Sarracenae, quibus ex morte Regis dolor non fictus ob venerat saccis operlae, passis crinibus et die noctumque turmatim incedentes ancillarum praecunctae moltitudine, totam civitatem ululatum complebant ad plusata tympana cantu flebilis respondentes" ${ }^{26}$

Un dolore ritualmente espresso che costituisce un dettaglio non trascurabile, capace di incidere la memoria collettiva. Tanto che nemmeno la malevola critica di Falcando lo può ignorare. Una contraddizione in vero, che lascia una suggestione più forte ed apre a presunzioni, quale quella concernente un'effettiva partecipazione al dolore per la regia dipartita da parte di una più ampia collettività.

Un dolore, quello delle donne, che rientra fra i motivi tipici delle narrazioni concernenti i riti funerari, anzi è loro prerogativa esclusiva, perché come afferma Malaterra: "foeminis lamenta permittitur". ${ }^{27}$ Un "obbligo simbolico" quello affidato alle donne, che oltre la scandita suddivisione delle relazioni fra i sessi, dimostra una certa verosimiglianza della narrazione, almeno nel riportare quei comportamenti ritualizzati nell'affrontare l'evento morte. Si ravvisa una materia su cui difficilmente si può fare opera di mistificazione. ${ }^{28}$ Questi si preoccupa pure di riferire alcuni dettagli come le lacrime ed i capelli sciolti, quali espedienti che rientrano nella tradizionale esternazione del dolore; descrizione che corona con l'espressione "non fictus".

Siamo di fronte a suggestioni e spunti che vanno considerati per quel che sono: meri indicatori. Essi non rendono giustizia ad un dolore corale, "insufflato" e persino indotto dal tempo mesto del lutto di Stato. La proposizione di un modello consueto con la sua teatralità di gesti non può far altro che avvalorare la percezione di un atteggiamento condiviso dalla collettività; esso sembra rispecchiare persino sentimenti piuttosto "uniformi". Registra insomma l'eco di uno stato d'animo, stimolato dagli stratificati espedienti che caratterizzano il tempo del lutto. Il dolore richiesto alla classe dirigente può persino persuadere il popolo, quasi coartarlo nella cerimonia e spingerlo sommessamente ad esprimersi secondo un formulario proprio e condiviso da tutti i partecipanti al rito funesto.

\section{La formositas e la magnificentia del re: la perfezione autocratica e la sua 'ga- ranzia' materiale}

Una penuria di fonti che si riscontra anche per Guglielmo II. La letteratura che commemora il suo decesso appare molto attenta alla fenomenologia del dolore ed all'emotività del lutto, ma sembra quasi ignorare la descrizione delle insegne e dell'abbigliamento

\footnotetext{
26 Ibidem.

27 Gaufredus Malaterra, De rebus siculis, II, c 46.

28 Salvatore Tramontana, L'effimero nella Sicilia... , pp. 33-34.
} 
che il sovrano riveste nella morte. Tuttavia deve osservarsi come tutte le fonti sembrano concordare su un dettaglio particolare: lo 'splendore' di Guglielmo defunto, che perdura anche nella morte e si pone a contraltare della "venustas" del Guglielmo bambino, perché in entrambi i casi è la sua bellezza ad ottenere "gratiam et favorem omnium". ${ }^{29}$ Un indizio dunque. Una bellezza, quella dei re, che costituisce un locus dell'apparato encomiastico ed è essa stessa una formula-base per le descrizioni del sovrano pro tempore, secondo il binomio già adoperato dall'Anonimo Normanno. Questi nell'enucleare le caratteristiche del condottiero perfetto non può far a meno di rappresentarlo come espressione di "gloria" e "bellezza". ${ }^{30}$ Una bellezza nella morte quella di Guglielmo II, che la morte stessa non può far venir meno e come tale è descritta puntualmente da Pietro da Eboli:

"Obitus Wilelmi secundi formosi regis Sicilie. Post obitum, formose, tuum, que sceptra gubernet et regat, ex proprio sanguine prole cares (...). Quis novit secreta tue purissima mentis? Quod tua mens loquitur, mundus et ipse taces....". ${ }^{31}$

Pietro ha a presentare alla sua audience un'alterazione del codice di rappresentazione del sovrano. Propone uno stridente ossimoro fra la rigorosa inerzia del corpo e della mente del re deceduto avverso la sua straordinaria bellezza, che non è nemmeno sfiorata dalla morte, quale 'garanzia' dell'ultra-vita. Pietro non parla della mera bellezza fisica, ma offre piuttosto un'evocazione dello splendore della dignità regia che viene enfatizzata nella prothesis.

Questa formositas non può ignorare il ricco corredo di simboli che rende i re quel che sono. Siamo così di fronte ad un sommesso indizio, ma sufficiente a far nascere ragionevoli presunzioni circa la qualità dell'abbigliamento adoperato per l'occasione. Sicché la formositas può essere persino liquidata quale sottoprodotto della veste adeguata al rango. L'abbigliamento si pone poi come 'catalizzatore' nei processi di ricognizione della bellezza regia da parte dei partecipanti al rito.

Il binomio veste adeguata-bell'aspetto costituisce un topos letterario e pertanto Pietro da Eboli non inventa nulla. Diversamente la kaiserkritik si suole giovare di un preciso espediente constante nel binomio trascuratezza dell'abito-bruttezza. Il logorio delle vesti viene così associato ad un aspetto fisico poco curato, se non proprio turpe. È a tal riguardo paradigmatico il locus estrapolato da Liutprando nella Relatio de legatione constantinopolitana, che nel criticare Niceforo Foca procede a demolire l'immagine istituzionale, enfatizzando sia le misere vesti, che la parvenza estetica, assimilandolo ad una "talpa" ed a un "etiope".

\footnotetext{
29 Falcandus, Liber de Regno Siciliae, 89, XXV; Salvatore Tramontana, L'effimero nella Sicilia... , pp. 26-27; 33-34.

30 Glauco Maria Cantarella, "Il pallottoliere della regalità:..., pp. 29-44.

31 Petrus De EBuLo, Liber ad honorem Augusti sive de rebus siculis carmen, particula XXIV; domine coacta descensio, Particula II, 35-40.
} 
Siamo di fronte ad una trappola della retorica del potere. Il martellante richiamo alla formositas, costituisce una formula di sintesi, che esprime in modo egregio una realtà ideale, riassumendo con sinteticità ed efficacia quelle molte parole che dovrebbero essere spese per descrivere lo splendore di un re.

L'evocazione della bellezza regia ha poi un carattere apologetico rispetto all'istituzione e specie avverso il successore, giacché Guglielmo diviene il re 'ideale' "nell'attualità della pienezza della sua perfezione". ${ }^{32}$ Egli non viene consegnato al ricco sepolcro per "marcire", ma assurge ad uno dei tanti 're nascosti' ${ }^{33}$ La bellezza al contempo diviene il 'marchio' per eccellenza della 'perfezione autocratica'. ${ }^{34}$ Un'ultra-vita, quella di Guglielmo II, che si percepisce piuttosto come apoteosi, laddove il vuoto lasciato dal decesso viene riempito dallo sfolgorio della sua formositas, quale 'sigillo della perfezione personale'. Ciò lascia apprezzare la totalità della pienezza della legittimità istituzionale, che in lui si incarna. Una percezione che ovviamente è resa più immediata dalle vesti preziose e dalla profusione dell'oro.

Siamo di fronte ad un'operazione quasi matematica, "abacista" potremmo dire, quasi un "calcolo" al "pallottoliere", quale sforzo apologetico estremo posto in essere dal retore a favore della monarchia locale. ${ }^{35}$

Ma vi è di più. Quella che Pietro da Eboli pone in essere è la falsificazione di un codice e nulla più di un altro trucco del narratore. ${ }^{36}$ Una falsificazione che si consuma in un'artificiosa bellezza capace di sopravvivere nella morte, quale parossistico locus del perdurare della condizione regia oltre il decesso. Artificiosa perché si fonda su un tentativo volto a dare per acclarato l'assorbimento di una delle formule retoriche della panegiristica entro lo schema dell'epitaffio.

Siamo di fronte ad una figura di forte impatto narrativo, che rientra nella tradizione della regalità cristiana sin dalla Vita Constantini. Quello speculum che nel delineare il principe perfetto riconosce l'indispensabilità della bellezza, suggerendo il suo ruolo di garanzia materiale rispetto alla legittimità dell'esercizio del potere. ${ }^{37}$ Un locus in verità molto più antico, che risale alla tradizione ellenistica ed iranica, laddove la bellezza del sovrano è pegno ed espressione esterna di un ordine in sé perfetto. ${ }^{38}$

Indi per cui il paradosso può essere serenamente assorbito entro l'alea della tradizione. La proposizione di questo codice non va liquidata come una delle tante altre possibili

\footnotetext{
32 Glauco Maria Cantarella, "Il pallottoliere della regalità:..., , pp. 29-44.

33 Ibidem.

34 Ibidem.

35 Ibidem.

36 Ibidem.

37 Eusebius Caesarensis, Vita Constantini, IV 69, 1; 70-71; Javier Arce, Funus imperatorum, Asgari, Madrid, 1989; Javier ARCE, "Morte e apoteosi del principe. Imperatori divinizzati", Aurea Roma. Dalla città pagana alla città cristiana, S. Ensoli y E. La Rocca (edd.), L'Erma Di Bretschneider, Firenze, 2000, pp. 244-248; Simon R. F. Price, Rituals and Power. The Roman Imperial Cult in Asia Minor, Cambridge University Press, Cambridge, 1994, pp. 75-78; Sabine G. MacCormack, (tr. It.), Arte e cerimoniale nell'antichità, Einaudi, Torino, 1995, pp. 169-175.

38 Glauco Maria Cantarella, "Il pallottoliere della regalità:..., , pp. 29-44.
} 
combinazioni del "pallottoliere della regalità", ma risponde ad un collaudato schema di cui costituisce il fiore dei 'fiori della retorica'. Si raffronta così un uso artificioso e cosciente del codice che si muove entro i limiti d'applicazione dello stesso. Un esercizio retorico che palesa la sua natura stessa: quello di codice squisitamente politico, sempre valido e pertanto pronto all'uso. ${ }^{39}$

Anche la formositas trova il suo contraltare: esso si rinviene nella magnificentia regia, che assorbe la bellezza ed amplia la serie dei significanti che questa può evocare. Perché essa si pone al contempo come 'garanzia' materiale dell'ultra-vita del sovrano.

La magnificentia diviene così un espediente retorico che viene fatto proprio dal componimento sulla dipartita di Guglielmo II opera di Riccardo di San Germano. Qui si dichiara apertis verbis che la magnificentia, quale clausola materiale posta a salvaguardia dell'eternità dell'istituzione, è capace di "insufflare" la suggestione che il defunto Guglielmo sia ancora vivo. Un'espressione che non è senza conseguenze sul piano retorico, ma anche su quello protocollare.

Questi afferma:

\author{
Rex Guillelmus abiit, \\ non obiit. \\ Rex ille magnificus, \\ pacificus, \\ cuius uita placuit \\ Deo et hominibus:
}

Eius semper spiritus

Deo uiuat celitus. ${ }^{40}$

Riccardo arricchisce il racconto di tutta una serie di espedienti già propri del protocollo funesto della corte costantinopolitana, quali soluzioni che affondano nella dottrina del potere romano e romano orientale. Fa così ricorso ad un espediente approntato per la prima volta alla morte di Augusto e sperimentato con successo per molto tempo, tanto che viene perpetuato nelle cerimonie funebri per la dipartita di Costantino: agire innanzi al sovrano come se fosse vivo. ${ }^{41}$ Siamo allora di fronte a più di un 'motivo errante della regalità', che viene utilmente mutuato dalla corte palermitana.

La veste preziosa a ragione può convincere a porre in essere a favore del defunto tutte quelle cortesie istituzionalizzate dal protocollo; in sua assenza l'espediente orchestrato dalla 'macchina' di corte andrebbe a vanificarsi. Anche questa soluzione costituisce a sua volta un codice, riconducibile ai primi tentativi d'incardinamento in

\footnotetext{
Ibidem.

40 Richardi De Sancto Germano Notarii Chronica, vv. 1-16-17.

41 Svet., Aug. 101; Dio Cas., LVI, 33, 1; Tac., Ann. I, 8, 1.
} 
Sicilia dei germi della dottrina del doppio corpo del sovrano. ${ }^{42}$ Una teoria che rende più comprensibile l'ulteriore sforzo retorico posto in essere dal narratore, mentre il codice si rivela essere una delle tante trappole poste dalla retorica del potere. Una trappola che non è null'altro se non una falsificazione della realtà. Ciò permette di eseguire in quel tempo straordinario tutte quelle manovre necessarie per garantire la continuità dell' istituzione monarchica.$^{43}$ Un esercizio politico insomma, che vale per quel che è e richiede a chi lo usa la capacità di saper padroneggiare i codici e il loro contrario. Si raffronta un'altra di quelle combinazioni possibili, il cui inserimento nella narrativa si giustifica tutto in una prassi tradizionale.

Il ruolo fondamentale svolto dalla veste viene così dimostrato dall'evocazione del codice comportamentale e dalla fictio. Ciò lascia presuppore che lo stesso sia abbigliato con vesti adeguate, che devono suscitare precisi sentimenti, timore perfino, verso colui da cui non ci si può più aspettare nulla. Si può così dire che la veste corrispondente al rango può ottimizzare questa finzione e coartare i partecipanti del rito funebre, obbligando a perseguire determinate condotte che neutralizzano gli effetti della dipartita. Diversamente, una prothesis sfornita di un'adeguata veste metterebbe in dubbio il carisma del sovrano stesso, cosa che implica l'impossibilità di sostenere ciò che a tutti è palese, oltre ogni trappola della retorica. Una scelta vestiaria indecorosa lede non solo la memoria del defunto, ma piuttosto l'istituzione, che viene privata di uno degli strumenti più immediati per la comunicazione dei suoi valori.

L'abbigliamento appare così funzionale alle strategie di auto-rappresentazione e costituisce uno degli strumenti indispensabili dell'apparato della caducità. Nonostante ciò si oppone ad esso e nel momento in cui lo nega, non fa altro che ribadirne l'effettività e l'efficacia sul piano delle interrelazioni sociali. Tale falsificazione della realtà presuppone allora che il sovrano si presenti nella prothesis in tutta la sua magnificentia e pertanto la precisa scelta semantica funge da puntuale indicatore, che fa propendere ancora per il perdurare di una cosciente utilizzazione della veste come segno. Una presunzione dunque, ma pur sempre capace di colmare almeno parzialmente quel vuoto che le fonti narrative lasciano a riguardo di questo specifico utilizzo sociologicamente orientato. Non gli rende nemmeno giustizia la miniatura alla Carta 4(97)a del Cod. 120 , ora conservato alla Biblioteca di Berna. Qui si intravede l'efebico volto coronato di Guglielmo II, che veste la consueta tunica blu cobalto, mentre viene avvolto da un drappo dal colore giallo paglierino, probabile allusione all'oro, quale unico dettaglio prezioso che conferma la magnificenza della prothesis. Tuttavia l'impiego del blu per la tunica funebre offre un sommesso indicatore dell'uso dei vestimenta regalia per la prothesis, un indizio che costituisce una presunzione, non sufficiente a convincere circa un effettivo impiego.

\footnotetext{
42 Ernst Kantorowicz, I due corpi del sovrano, L'idea di regalità nella teologia politica medievale, Einaudi, Torino, 2012.

43 Glauco Maria Cantarella, "Il pallottoliere della regalità:..., , pp. 29-44.
} 
FIG. 2.

La morte di Guglielmo II, miniatura, Carta 4(97)a, Cod. 120, Biblioteca di Berna.

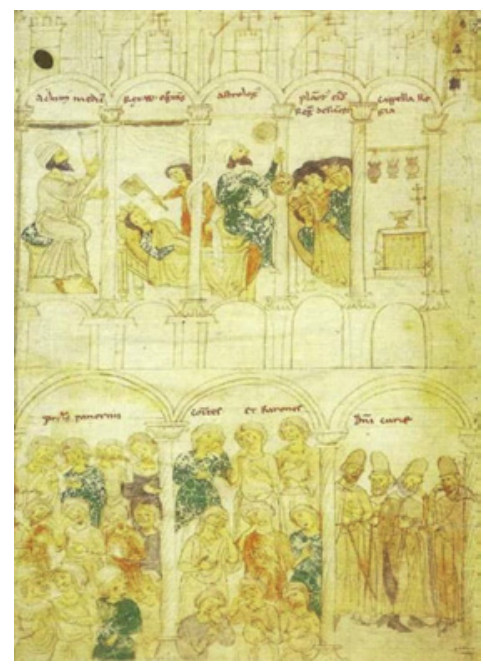

FIG. 3.

Il lutto per la morte di Guglielmo

II, miniatura, Carta 5(98)a, Cod. 120, Biblioteca di Berna.

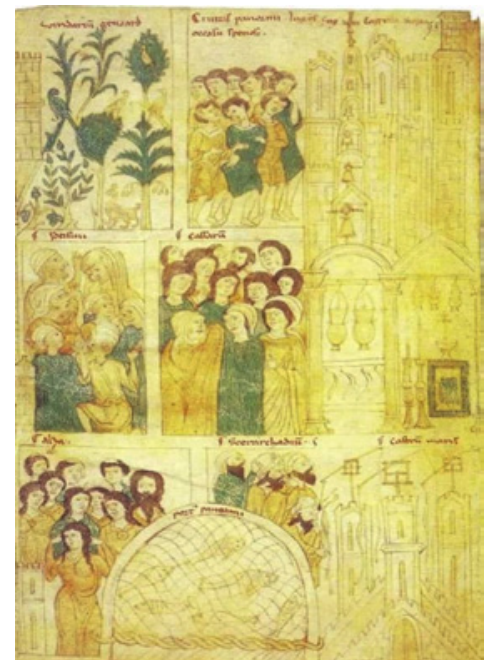

7. La veste funebre di Enrico VI e la cromia della regalità: quale eloquente evidenza tessile

Il valore sociale della veste può essere meglio apprezzato se si considera l'inumazione di Enrico VI nella cattedrale palermitana. L'ispezione del 1781 offre una puntuale descrizione del corredo funebre che accompagna nella tomba il sovrano. Ciò lascia considerare un gusto per la significatività dell'abbigliamento, che viene ribadito innanzi all'evento morte attraverso una mise adeguata all'officio imperiale. Un gusto tutto riconducibile alla classe, che non può prescindere dal tessuto prezioso, dall'abito dal taglio ricercato e riccamente decorato per manifestare il rango. Un abito che anche nel buio del sepolcro deve ottemperare a quella funzione dell'ostentazione dell'abbondanza regia, che è cifra del peculiare status ${ }^{44}$ Un gusto che si riflette anche nei colori. Compare difatti il cremisi, quale adeguato sostituto della porpora, specie se si considera la povertà semantica del Medioevo latino che non conosce termini adeguati per lasciar percepire le differenti sfumature di tonalità assimilabili. ${ }^{45}$ Viene utilizzato pure il verde, cromia che in Occidente si oppone ed è alternativa alla porpora stessa, perché capace di evocare la sua variante più pregiata: il "semimela", la

44 Salvatore Tramontana, Vestirsi e travestirsi... , pp. 45-47; 52.

45 Fridericus II, De arte venandi cum avibus, VII; Salvatore Tramontana, Vestirsi e travestirsi... , pp. 94-95. 
cosiddetta "porpora proibita", oggetto di commercio di veneziani e amalfitani. ${ }^{46}$ Una tinta adoperata per il manto imperiale utilizzato da Enrico IV per la sua incoronazione romana, almeno stando a quanto attesta Benzone d'Alba. ${ }^{47}$ Tono che sembra divenire espressione di giocondità e di lussuria e, pertanto, viene preferito da Federico e dal figlio Manfredi come attesta Villani. ${ }^{48}$ Nonché si ravvisa l'uso del turchino, che con la sua alea di senso allude sempre alla monarchia divina. Tonalità ordinate secondo una scala condivisa di valori, che manifestano l'intrinseca dignità del defunto. La loro presenza dichiara il non venir meno dello status imperiale con la morte, diventando pegno dell'ultra-vita del governante, che dal sepolcro ed in modo invisibile continua l'esercizio del potere. Una continuità ribadita dall'oro come ulteriore 'clausola di salvaguardia' posta a garanzia del rango e quale espressione di un gusto di classe condiviso, che esige il dettaglio prezioso; una cromia che appare indispensabile nei processi ricognitivi di status. L'uso dell'oro, fatto proprio dalle elaborazioni della dottrina del potere laico, nel momento in cui si oppone ai dispostivi sulla caducità del singolo rappresentante pro tempore ha a negarli, perché questo è pegno del suo sopravvivere come corpo politico a garanzia della continuità istituzionale. ${ }^{49}$

Tutti i colori si apprezzano però nella tinta data loro dal tempo, uno stato peggiorato dai problemi di conservazione dell'intensità del tono dovuti al procedimento di tintura, che come noto non è efficace sul lungo periodo. Ciò non permette di percepire l'effettivo impatto dell'opera al momento dell'inumazione e viene così meno la possibilità di apprezzare la fatica degli artigiani che hanno intessuto le stoffe e le hanno decorate. Un discorso sulla caducità che si concentra pure sul corpo, facendone uno dei codici con cui viene attualizzato. Un paradosso insomma: nel momento in cui si ammette la transitorietà del defunto sovrano, non si vuol far altro che ribadire l'eternità istituzionale. Il corpo adeguatamente abbigliato, esposto nei riti che precedono la consegna al sepolcro, diviene il primo dei luoghi in cui è possibile affermare la nuova elaborazione dottrinale avverso la censura della morte, mentre la protheis costituisce un momento privilegiato per somministrare tale idea al pubblico. L'ostentazione rituale della salma diventa pure l'occasione per attestare la pregevolezza del lignaggio, il cui valore si apprezza attraverso l'esibizione dell'abbigliamento che lo riveste. Una finalità ricognitiva quella della veste, che si esplica nell'immediatezza ed attraverso l'impatto visivo, quale momento della comprensione totalizzante, capace di fornire tutte le informazioni essenziali sulla collocazione nella gerarchia sociale.

\footnotetext{
46 Rocco Antonio CARILE, Produzione e usi della porpora nell'impero bizantino, Istituto Veneto di Scienze, Lettere ed Arti, Venezia, 1998, p. 246; Glauco Maria Cantarella, "Le basi concettuali..., pp. 199-201.

47 Benzo von Alba, Ad Henricum imperatorem IV, Libri VII, LVIII; Glauco Maria Cantarella, "Le basi concettuali..., pp. 213-219.

48 Giovanni Villani, Nuova Cronica, 1, VI, c. 46; Salvatore Tramontana, Vestirsi e travestirsi... , p. 98.

49 Giovanni Tabacco, Le ideologie politiche del Medioevo, Einaudi, Torino, 2000; Dieter Mertens, Il pensiero politico...; Glauco Maria Cantarella, Medioevo. Un filo... ; Glauco Maria Cantarella, "Le basi concettuali... , pp. 193-207; Glauco Maria CAntarella, "Divagazioni preliminari”... , pp. 9-24.
} 
Non meraviglia allora che la cura per il dettaglio prezioso e per i materiali ricercati delle vesti funebri dei sovrani trovi ampio spazio pure nella relazione di Rosario Gregorio. Questi tuttavia pone in essere più una sommessa disquisizione ideologica, che l'asettica verbalizzazione dello stato di conservazione delle evidenze rinvenute nella tomba. Una descrizione che non rispecchia meramente l'oggetto in sé, ma piuttosto illumina circa la percezione condivisa al tempo dell'autore del valore e della significatività del capo sul piano culturale e sociale; una pregevolezza comunque quantificabile con i criteri dell'economia. Si ripropone una velata strategia che ricorre al principio dell'abbondanza propria dei sovrani e ne somministra efficacemente gli etimi; un criterio quello dell'opulenza che è indispensabile se si ha a dar conto della dignità imperiale.

La descrizione di Rosario Gregorio riferisce così puntualmente dell'abbigliamento con cui viene ornato l'imperatore Enrico VI al momento in cui è consegnato alla morte ed ai suoi riti:

"Tutto il corpo, secondo quel che ne potè apparire, era coperto di un drappo di seta di color pendente al giallo: e le sue estremità all'altezza di un palmo sono fregiate di drappo color cremisi, con oro intessuto a vario disegno. Avea una cintura di seta sciolta, e di tratto in tratto in più nodi legata, di dietro ha essa involto un pannolino, ed è annodata dinnanzi: a ciascun dei suoi lati sono attaccati molti cordoncini di seta, tessuti di due colori verde e cremisi, ed entrando essi in alcuni occhielli delle brache, le tenevano alla cintura legate. Dal nodo dinnanzi pendono due frange di seta larghe tre dita, tessute a spigha di tessuto scauratino, giallognolo, e turchino, e sono nelle estremità sfioccate. Sul ventre erano sparse più ciocchette di capelli di vario colore. Le coscie e le gambe eran vestite di drappo, e formava in uno calze e calzoni. Vicino ai piedi era l'altro guanto ed una beretta, ossia mitra imperiale di colore giallo. Ha essa un fregio d'oro ornato di arabelli, e scudetti, dentro $i$ quali sono intessute alcune lettere arabe di colore nero, e pendevanle dalla parte di dietro $i$ due bendoni. Era calzato di belle scarpe. Il tomajo di esse è di seta lavorata a certi compassi di oro e di perle, ed il suolo è di sughero foderato di drappo di vario colore. Non si trovò ne spada né corona" ${ }^{50}$

Eppure deve osservarsi che questa descrizione per quanto puntuale è solo parziale e si limita a 'fotografare' lo stato dei fatti ad una precisa data. Il sarcofago difatti è stato aperto diverse volte ed il cadavere sicuramente manomesso. Ciò spiegherebbe ad esempio l'assenza della corona e della spada, elementi essenziali nel corredo di un sovrano. L'arma difatti non manca mai nell'inumazione approntata secondo il mos nobilium. È nota l'ispezione della salma posta in essere dal viceré Ferdinando de Acuña nel 1490, allorquando viene arrestata dal tumulto popolare. Cosa che non ha

50 Rosario Gregorio, Discorsi intorno la Sicilia, Pedone, Palermo, 1923, pp. 25-26. 
garantito dall'asportazione di pezzi del vestiario o del corredo più o meno rilevanti, alterando l'originario stato del corpo e la sua composizione. ${ }^{51}$

Attraverso le stampe poste a corredo della descrizione dei sepolcri palermitani di Daniele è pure possibile apprezzare la qualità dei decori e specie il fine ricamo a motivi zoomorfi e vegetali di un frammento della veste di Enrico. ${ }^{52}$ Questo presenta l'alternarsi entro racemi di coppie affrontate di volatili e cervi accostati a tergo, che si dispongono su registri alternati. Altrettanto raffinati appaiono i decori della mitra clericalis che accompagna la sepoltura, laddove si dispongono i sottili rami di acanto provvisti di fiori a creare dei clipei di diversa grandezza, tanto da dar spazio ad iscrizioni in caratteri cufici e piccole croci..$^{53}$

FIG. 4.

\section{Frammento della veste di Enrico VI, xilografia da F. Daniele, I reali sepolcri di Palermo riconosciuti ed illustrati, Palermo, 1784, Tav. F.}

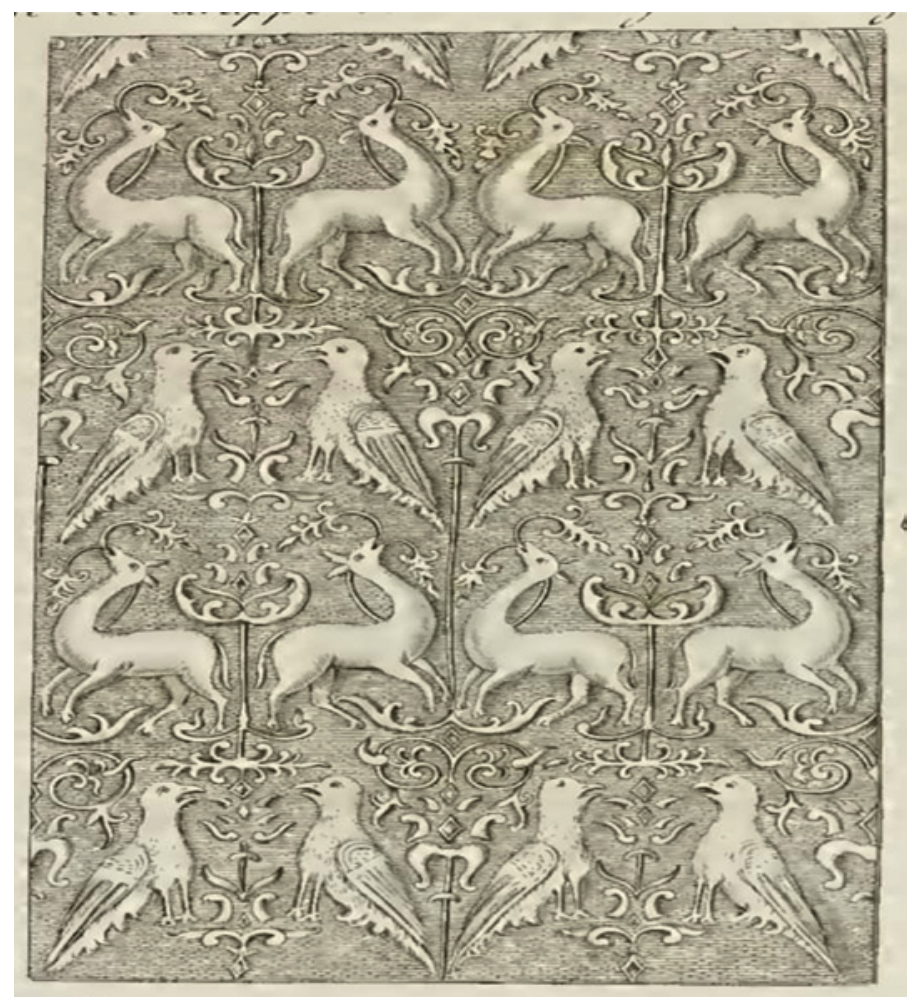

1 T. Fazzello, De rebus siculis, Pedone, Palermo, 1833, II, 1; IX, c. 10.

52 Rosalia Varoli Piazza, "Per un approccio..., pp. 154-155.

53 Jeremy Johns, "Le iscrizioni e le epigrafi in arabo", Nobiles Officinae: perle, filigrane e trame di seta dal Palazzo Reale di Palermo, M. Andaloro (ed.), Maimone, Catania, 2006, pp. 119-131. 
FIG. 5.

\section{Ricami posti a decoro della mitra clericalis di Enrico VI, xilografia da F. Daniele, I reali sepolcri di Palermo riconosciuti ed illustrati, Palermo, 1784, Tav. H.}

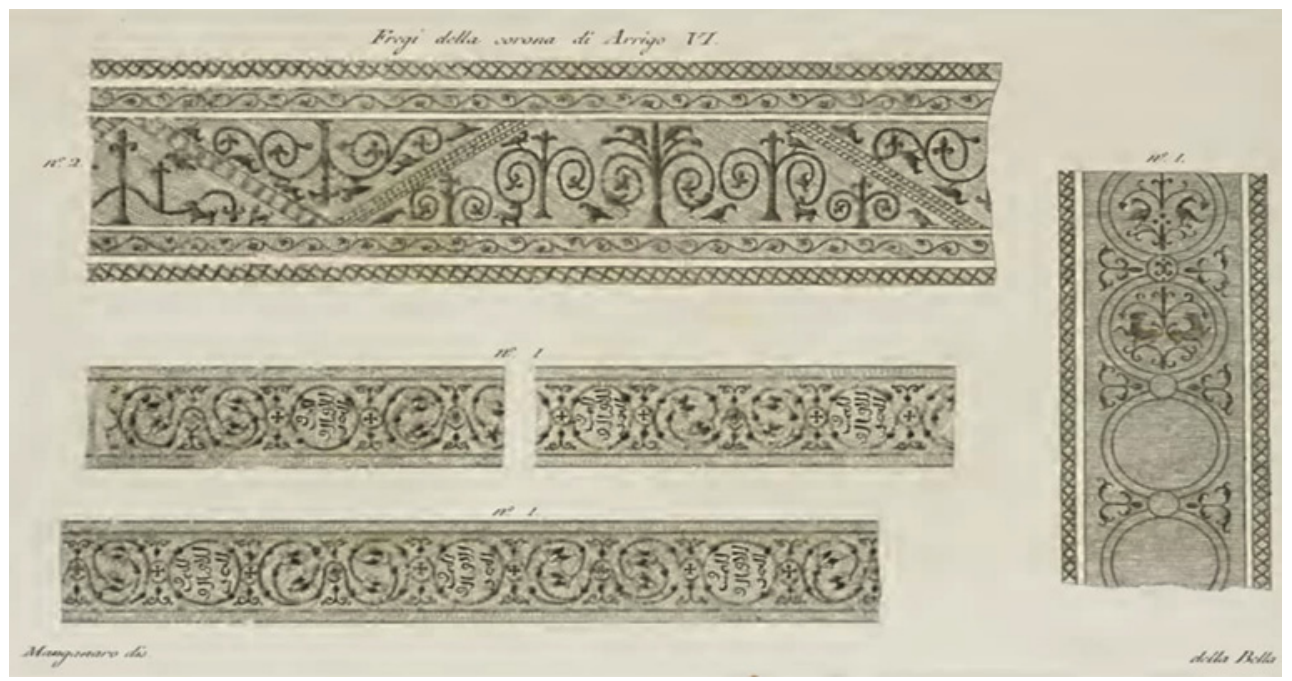

8. Veste regale o abito monastico? L'abbigliamento funebre di Federico II quale 'clausola di salvaguardia' avverso le elaborazioni dell'apparato di caducità del sovrano

Le fonti concernenti la morte di Federico II sono le più prodighe di dettagli. ${ }^{54}$ Questi, allorché vivo, ha inciso l'immaginario collettivo con la rituale ostentazione

\footnotetext{
54 Percy Ernst Schramm, Kaiser Friedrichs II. Herrschaftszeichen, Vandenhoeck \& Ruprecht, Goettingen, 1955; Raffaele Iorio, "Umanità disumana di Federico persona e personaggio", Federico II. Mezzogiorno, Atti del Convegno Internazionale di Studio, Potenza-Avigliano-Castel Lagopesole- Melfi, 18-23 ottobre 1994, C.D. Fonseca (ed.), Edizioni de Luca, Roma, 1999, pp. 701-748; David Abulafia, Federico II. Un imperatore medievale, Einaudi, Torino, 1993; Mirko VAGNonI, Federico II allo specchio. Analisi iconografica e politico funzionale delle sue raffigurazioni, Tesi di Laurea in Storia, Università degli Studi di Siena, Anno Accademico 2003-2004; Mirko VAGNonI, "Il significato politico delle caratteristiche iconografiche di Federico II di Svevia", Iconographica. Rivista di iconografia medievale e moderna 5 (2006), pp. 64-75; Mirko Vagnoni, "La legittimità e la sacralità imperiale di Federico II di Svevia", Tabulae. Centro Studi Federiciani 18 (2006), pp. 127-169; Reinhard Elze, "Le insegne del potere"..., pp. 45-51; Reinhard Elze, "Tre Ordines per l'incoronazione di un re e di una regina del regno normanno di Sicilia", Atti del Congresso Internazionale di Studi sulla Sicilia Normanna, Palermo, 4-8 dicembre 1972, Istituto di Storia Medievale Università di Palermo, Caltanissetta-Roma, Sciascia, 1973, pp. 438-459; Franco CArdinI, "Federico II. La memoria e il mito", Federico II. Immagine e potere, Catalogo della Mostra, Bari, Castello Svevo, 4 febbraio-17 aprile 1995, M. S. Calò Mariani y R. Cassano (edd.), Marsilio, Venezia, 1995, pp. 453-454; Fulvio Delle Donne, Il potere e la sua legittimazione: letteratura encomiastica in onore di Federico II di
} 
dell'abbondanza, una suggestione a corollario delle strategie di auto-rappresentazione imperiale, che non può essere negata dalla cesura imposta dall'evento morte. Siamo così di fronte ad uno dei tanti obblighi del rango, specie di quello imperiale, che la morte rende ancor più stringente ed inemendabile. Tuttavia deve ravvisarsi che la capacità affabulatoria della consueta ostentazione d'abbondanza ha il suo contraltare nel bilanciamento di interessi fra l'esternazione di una prerogativa riconducibile alle tecniche volte alla spettacolarizzazione ed un accidente della storia evenemenziale di Federico: la scomunica inferta dall'acerrimo nemico Gregorio IX.

La forza della scomunica costituisce un dettaglio di certo non trascurabile, che interessa non tanto le relazioni Chiesa-Impero, ma piuttosto la macrostruttura del sistema sociale ed i rapporti entro l'istituzione imperiale, la nobiltas nei suoi diversi gradi e la gente comune. Un accidente che obbliga la 'macchina statale' di produzione del consenso ad un lavoro più alacre, per ridurne l'impatto sull'opinione pubblica, specie quella della gente comune. ${ }^{55}$ Anche perché il morire sotto scomunica non è senza conseguenze sia per l'ultra-vita del sovrano, sia per la continuità istituzionale.

Una 'macchina' che preferisce muoversi entro espedienti consueti e sperimentati con successo, quali la spettacolarizzazione dell'evento. Un dispendio di risorse da devolvere all'effimero, che usa come strumento principe il fasto degli abiti ed i dettagli preziosi, quali garanzie del perdurare dell'eccellenza del rango rispetto alla censura ecclesiastica. Questi espedienti infatti sono ritenuti capaci di far dimenticare al popolo la situazione eccezionale in cui l'evento morte si consuma. I dettagli d'opulenza sembrano dotati di una forza 'normalizzante': quella dell'ordinarietà del già visto; essa si correla all'ossequio di una tradizione ancestrale legata ai costumi funerari, cosa che carica la messa in scena di forza rassicurante. Una straordinaria 'normalità' dunque, che s'imprime nella fantasia degli astanti del corteo funebre, anche in quelli abbienti, come il cavaliere Spinelli di Giovinazzo.

Una 'normalità' che paradossalmente esige un'attenzione al dettaglio. Questa diventa ancora più 'morbosa' se si considera la sommessa evocazione nelle fonti della presenza dei capi, specie se confrontata col silenzio di una gran parte della letteratura precedente. La cura nel registrare gli oggetti che compongono il corredo funebre di Federico non può voler dire altro: l'abbigliamento è un efficace segnacolo di quel potere, che

Svevia, Nuovi Segnali, Arce, 2005, pp. 59-98; Carlrichard BRÜHL, "Federico II: personalità di un sovrano", Federico II e il mondo Mediterraneo, P. Toubert y A. Paravicini Bagliani (edd.), Sellerio, Palermo, 1994, pp. 17-30; Maria S. Calò Mariani, "I fenomeni artistici come espressione del potere", Potere, società e popolo tra età normanna ed età sveva (1189-1210), Atti delle Quinte Giornate Normanno-Sveve, BariConversano, 26-28 ottobre 1981, Dedalo, Bari, 1983, pp. 215-250; Maria S. Calò Mariani, "L'arte al servizio dello Stato", Federico II e il mondo Mediterraneo, P. Toubert y A. Paravicini Bagliani (edd.), Sellerio, Palermo, 1994, pp. 123-145; Maria S. Calò Mariani, "Immagine e potere", Federico II. Immagine e potere, Catalogo della Mostra, Bari, Castello Svevo, 4 febbraio-17 aprile 1995, M. S. Calò Mariani y R. Cassano (edd.), Marsilio, Venezia, 1995, pp. 39-43.

55 Giulia Barone, "La propaganda antiimperiale nell'Italia federiciana: l'azione degli Ordini Mendicanti”, Federico II e le città italiane, P. Toubert y A. Paravicini Bagliani (edd.), Sellerio, Palermo, 1994, pp. 278-289. 
l'imperatore conserva nella morte. Esso poi si pone quale 'garanzia' avverso gli effetti invalidanti della scomunica. E se il rango viene confermato dall'equazione bello-ricco di un abbigliamento indossato nelle occasioni di socialità, allo stesso modo l'eccellenza dei suoi materiali e dettagli costituisce una 'clausola di salvaguardia' materiale. La veste configura così un'eloquente affermazione della legittimità dell'esercizio dell'Impero.

Alla base della velleità profusa nella descrizione si riconosce dunque un ragionamento più sottile, fin troppo attento all'enucleazione di certi simboli dell'eccellenza. Una cura che lascia trasparire gli ulteriori significanti che la corte, nel momento in cui pone in essere la messa in scena volta al consenso, vuole effettivamente trasmettere. Siamo di fronte ad una prova provata della condivisione del valore della veste a riprova della sua efficacia verso un pubblico in progressiva estensione. Il fatto che questi dettagli siano registrati non dalla nobiltas, ma dal ceto medio-alto della provincia, si pone a puntuale dimostrazione del valore effettivamente percepito non solo da questa classe intermedia, che è in grado di leggere e può gestire lo strumento della scrittura, ma dalla massa. Un meccanismo di fascinazione connesso alla devoluzione di risorse volte all'effimero, che ammalia il popolo e lo persuade, perché questo non è affatto estraneo a quel senso di meraviglia suscitato dall'opulenza. Un sentire che ha maggior presa, se si considera la penuria atavica che attanaglia la classe infima. Una riflessione sugli etimi del potere e sui suoi mezzi di comunicazione che deve tenere in conto della sensibilità delle diverse classi. Ciò fa luce su alcune implicazioni non adeguatamente indagate nelle relazioni intercorrenti fra i diversi ceti dell'Occidente.

Uno spettacolo davvero, che attira la gente, anche quello Spinelli da Giovinazzo il quale offre alcuni dettagli interessanti sulla cultura materiale connessa alla fenomenologia del dolore: il velluto cremisi che copre il corpo di Federico trasportato in lettiga ad esempio. Costui tuttavia non fornisce maggiori dettagli sull'abbigliamento, ma si limita a riferire meramente quello che il suo occhio ha potuto vedere e quanto un qualunque avventore ha possibilità di notare al passaggio del corteo funebre:

\begin{abstract}
"Alli 13 che fo lo dì di Santa Lucia morio (...). Alli 28 dello mese passao lo corpo dello imperatore, che lo portarono a Taranto et io fui a Bitonto per vedere. Et andao in una lettica coperta di velluto cremisino, colla guardia de li Saracini a pede, et sei compagnie di cavalli armate, che come intravano le terre, andavano chiangendo l'imperatore e poi veneano alcuni baruni vestitii nigri, co' sindaci delle terre dello Reame (...)". ${ }^{56}$
\end{abstract}

La scelta di far rilevare i dettagli fastosi si riconduce ai concreti sforzi della corte e specie dei due uomini più vicini a Federico: Manfredi, fattosi proclamare Balio del Regno, che nella minorità di Corrado può fare le 'prove' da regnante, ed il vescovo di Palermo, che sul letto di morte assolve il sovrano dalla scomunica, attestando la veridicità della

56 A. Loparco (ed.), Matteo Spinelli, Diurnali, II, Cannone, Bari, 1865, pp. 62-63. 
contrizione e la penitenza effettuata. Quest'ultimo poi riempie quel ‘vuoto' creato dalla scomunica con l'autorità della propria funzione episcopale. Il 'vuoto' generato dallo 'scollamento' fra le due massime istituzioni del Medioevo, può essere riempito solo fino ad un certo punto dalla scelta di un preciso abbigliamento. Se ne deduce che la veste non offre una garanzia totale. Tuttavia non si può negare che l'opulenza ritualmente ostentata manifesta una rassicurante volontà.

La narrazione pone di fronte ad un complesso sistema di contrappesi ideologici, retorici e persino materiali, tanto che le stesse vesti posso essere considerate anche uno strumento ideale per sugellare l'azione di kaiserkritik, come negli effetti accade nell'opera di Villani. Siamo di fronte ad una materia duttile, che concerne non solo i discorsi sulla caducità di una delle "super-personalità" del Medioevo. Ci si approssima soprattutto ad un problema retorico, legato all'ambiguità dei termini di un'opera di convincimento, che pur di persuadere può dire tutto, ma anche il suo contrario. Un'ambiguità a cui la veste non si sottrae, perché ciò implica tutte quelle possibilità che rientrano in un'amplia alea di senso. Le vesti come strumenti altrettanto duttili si sottomettono poi alla forza dell'argomentazione, che aggiunge pregevolezza o la fa venire meno, mentre il narratore si muove nello spazio concesso dalle strutture mentali della cultura di riferimento per operare la critica.

Non meraviglia che questi possa procedere alla tabuizzazione della descrizione dell'abbigliamento indossato ed, al contempo, abbia a reintrodurre il tema della veste. Nella cronica del Villani difatti si ravvisa un'assenza di quei dettagli utili a ricostruire l'abito di Federico. Vi è solo un indicatore lessicale che può far intuire il rispetto del rango e l'uso di un corredo vestiario adeguato all'evento. La processione funebre viene velocemente descritta da un solo aggettivo: "nobilmente". Il lemma, con la sua carica semantica piena di significatività, lascia immaginare che i capi adoperati per Federico siano davvero adeguati al suo status imperiale.

Villani ha cura di introdurre un ulteriore dettaglio a coronamento della descrizione del rito funebre: menziona un presunto epitaffio dedicato all'imperatore, che evoca col suo ritmo incalzante quella "veste di virtù" della tradizione cristiana, che il sovrano scomunicato rivendica per sé. Una metafora, che molto ha da dire sulla significatività dell'abito e sul suo doppio valore ricognitivo. In ultima istanza esprime all'esterno niente più di quello che è uno stato interiore insieme alla dignità del rango. Una veste che Federico sembra indossare davvero grazie agli artifici di quei 'fiori della retorica' opportunamente adoperati. ${ }^{57}$ Uno stratagemma oratorio che gioca sul paradosso e nega l'esercizio delle virtù da parte dell'imperatore. Villani consapevolmente pone in essere una dicotomia volta a neutralizzare la significatività del ricorso alla metafora della "veste di virtù" da lui stesso introdotta, per farne mero oggetto di critica. Una contraddizione in termini dunque, che è seguita dalla stigmatizzazione dei peccati dello stesso sovrano, quale ulteriore contrappeso retorico dell'epitaffio. Mutua così loci dai florilegi della letteratura penitenziale per operare un bilanciamento dei valori e colpire

57 Glauco Maria Cantarella, "Divagazioni preliminari”..., p. 21. 
l'alea antropologicamente connotata della significatività della veste. Questo perché Villani sa bene, o per lo meno intuisce, che il modello puntualmente adoperato non ammette alternative. Il contro-bilanciamento allora si rivela quale necessità prima di tutto politica, poi retorica, che suggerisce argomentazioni con cui avvalorare il giudizio negativo, che condanna il Federico morente.

L'onnipotenza espressa dalla 'macchina' di corte, ostentata con sicurezza anche a dispetto della scomunica, merita i giusti contrappesi, quali gli accorgimenti retorici che devono stigmatizzare l'impasse in cui il sistema politico che regge l'Occidente è precipitato. Una serie di calmieri insomma, che rispecchiano tuttavia una visione parziale: quella della politica papale.

Si raffronta un altro 'fiore della retorica', volto a generare pathos narrativo. Un trucco dello storico dunque, che con disincantata ironia stigmatizza tutte le contraddizioni della teoria del potere statale e, al contempo, svela una delle 'trappole' consuete nella descrizione degli episodi della regalità declinati dalla fenomenologia del dolore. Questi, adoperando consapevolmente un preciso novero di codici in modo più o meno disinvolto, investe l'intero universo mentale concernete la potestà imperiale, denudandolo di tutte quelle espressioni di cortesia che si è soliti offrire ai sovrani. Una messa a nudo volta ad opporvi un'altra serie di codici, che costituiscono a loro volta una mistificazione dei fatti entro una visione politicamente orientata. Siamo di fronte ad un 'cambio di etichette' che nell'ottica di un'incisiva kaiserkritik manipola la memoria e tenta di investire una delle strutture che reggono il cosmo medievale. Un esercizio retorico di successo, perché in fin dei conti non fa altro che acclarare la vittoria del papato, che lascia l'istituzione imperiale indebolita e ciò anche a dispetto delle elaborazioni dei giuristi del XII sec. e federiciani in particolare.

Villani ha così a raccontare le vicende intorno alla morte di Federico:

"Nel detto anno MCCL, essendo Federigo imperadore in Puglia nella città di Fiorenzuola a l'uscita d'Abruzzi, si amalò forte (...). Avenne che agravando de la detta malattia, essendo collui uno suo figliuolo bastardo ch'avea nome Manfredi, disiderando d'avere il tesoro di Federigo suo padre, e la signoria del Regno e di Cicilia, e temendo che Federigo di quella malatia non iscampasse ofacesse testamento, concordandosi col suo segreto ciamberlano, promettendoli molti doni e signoria, con uno pimaccio che a Federigo puose il detto Manfredi in su la bocca, sì ll'afogò; e per lo detto modo mori il detto Federigo disposto dello 'mperio e scomunicato da santa Chiesa, sanza penitenzia, o nullo sagramento di santa Chiesa (....); e ciò fu il di di santa Lucia di dicembre, gli anni detti MCCL. E lui morto, Manfredi detto prese la guardia del reame e tutto il tesoro, e 'l corpo di Federigo fece portare e soppellire nobilemente alla chiesa di Monreale di sopra a la città di Palermo in Cicilia, e a la sua sepultura volendo scrivere molte parole di sua grandezza e podere, e grandi cose fatte per lui, uno cherico 
Trottano fece questi brievi versi, i quali piacquero molto a Manfredi e agli altri baroni, e fecegli intagliare nella detta sepultura, gli quali diceano:

Si probitas, sensus, virtutum gratia, census, Nobilitas orti possint resistere morti, Non foret extintus Federicus qui iacet intus." ${ }^{58}$

La narrazione incarna una critica che si colora dei toni dolenti della tragedia umana di Federico e della sua dinastia, la quale giunge all'auto-distruzione. Villani fornisce un contrappeso alla virtù laica o, almeno, alla pretesa dell'esercizio della virtù svolta non in unisono con la Chiesa. Una condanna assoluta, che costituisce l'espressione dell'abilità retorica dell'autore e soprattutto della sua capacità nell'usare quei codici propri del genere letterario.

Nel silenzio di Spinelli e Villani, ci si limita alla mera ricognizione di indicatori circa l'abbigliamento della salma e sulla loro scorta si operano delle congetture. Si può così postulare il rispetto anche nella morte delle scelte rappresentative fatte proprie da questo imperatore, che non ha mai disdegnato il lusso e lo sfarzo, perché confacenti all'istituzione che incarna.

Questo perché la narrazione, al di là delle velleità descrittive più o meno circostanziate, costituisce sempre una fonte ideologica: vuol rappresentare una realtà che va oltre la mera cronaca e non si limita a significare l'oggetto nelle sue qualità intrinseche. Il Villani in particolare si pone in questo sentire, mentre evoca la significatività delle condotte e ne spiega il valore politico, religioso e soprattutto culturale.

Maggiori dettagli vengono estrapolati dalla relazione di Rosario Gregorio, il quale offre una puntuale descrizione delle insegne che rivestono Federico e registra con cura la fattura dell'abbigliamento e delle insegne che lo accompagnano nel sepolcro, quali esemplari d'esclusività del rango:

"Era esso di ornatissimi vestimenti coperto. Nella testa, posata sopra di un cuscino di cuoio, aveva una corona aperta, i cui raggi di sottilissime laminette di argento dorato, erano ornati di perle e di pietre. Dal lato sinistro della testa era riposto il globo imperiale. Tre tuniche vestivano il cadavere. La prima pare un piviale di drappo lavorato, che si stringeva al petto cou un giojello di figura ovale di amatista incassata in oro, circondata da venti piccoli smeraldi, ed a quattro estremità di esso erano quattro grosse perle. La seconda, che è di drappo semplice, e senza niun lavoro, pare una dalmatica con maniche terminate con un gallone di oro largo quattro dita, ed era essa cinta da uno stretto gallone di seta, adornato di varie rose di argento indorato. La terza finalmente è un camice di lino il quale scendeva fino a coprire le cosce e le gambe, e lo cingeva un grosso cordone di lino aggrappato nel mezzo, e pendeva dall'uno dei lati. Si vide nel

58 Giovanni Villani, Nuova Cronica, 268, XLI. 
camice dalla parte sinistra sotto al collo ricamata una croce, e l'estremità del collo e delle maniche sono ornate di fregi a tre ordini, e nelle maniche il fregio maggiore è ornato a lettere cufiche. Le sue mani incrocicchiate posavano sul ventre, e in un dito della destra era un anello di oro con uno smeraldo. Dal fianco sinistro era posta la spada con la manica di legno attorno a cui erano attorcigliati serratamente sottilissimi fili di argento: tutto poi il guernimento è di argento indorato, e vi ha in esso tre anellini, dove entravano più cordoncelli di setanelle punte sfioccati. La sua cintura era un gallone di seta, tessuto stretto e serrato al disegno, di color cremisi che tira sul fosco, ed è ad esse appicato un fermaglio con assai altri fregi in argento indorato, nei quali venggonsi altri lavori. Era egli dalle coscie sino ai piedi vestito di panno, che parelino, e formava in uno calzoni, calze e pedali, si trovò calzato di stivaletti di seta, le cui scarpe erano nel tomajo ornate da un gallone dall'alto al basso e nel mezzo è tessuta una cerva: avevano esse gli sproni ciunti al di sopra con corregia". ${ }^{59}$

Non bisogna però dimenticare quanto riferisce Matteo Paris, che pare più attento al dettaglio dell'abbigliamento. Questi introduce quella dicotomia fra l'espediente della vestizione del saio dei cistercensi e il riscontro materiale delle imperialia insignia con cui è consegnato al sepolcro. Un'umile vestizione di cui l'autore favoleggia, quale segno esteriore utilissimo, atto a dimostrare la contrizione del cuore di Federico che gli vale pure la revoca della scomunica da parte dell'arcivescovo di Palermo. Un dettaglio che rende meno complessa la sutura dello 'scollamento' fra le due massime istituzioni. Ciò avviene nel marco della penitenza, quale atto rituale che non è certo una novità, ma ha precedenti autorevoli. Un'umiliazione altrettanto rituale giustificata su plurimi piani: quello sociale e della dicotomia tra laicato e sacerdozio, perché quest'ultimo è detentore di quella supremazia dovuta all'esercizio dell'immaginazione trasfiguratrice. Nonché quello politico e della 'ragion di Stato', che si nutre della speculazione concernente gli effetti della scomunica sul legittimo esercizio dell'Impero.

Al di là del locus retorico che la vestizione dell'abito monastico può evocare, l'abbigliamento in questo preciso contesto culturale assurge a mezzo di trasmissione di particolari valori. La veste diviene espressione di una pienezza di dignità dovuta all'adesione ad un preciso modello di vita, che è fatto proprio dall'ordine a cui la veste si riconduce, divenendo pegno del carisma di santità vantato dall'ordine stesso. $\mathrm{Al}$ contempo si pone quale 'clausola di salvaguardia' verso il morente, fungendo da prova provata di una pietà religiosa ritrovata. Si oppone poi avverso gli atti non troppo devoti di cui è solitamente incolpato. ${ }^{60}$

\footnotetext{
59 Gregorio Rosario, Sui reali sepolcri della maggior chiesa di Palermo, Dato, Palermo, 1781, pp. 711712 .

60 Salvatore Tramontana, Vestirsi e travestirsi..., p. 53.
} 
Eppure la menzione dell'abito monastico non deve meravigliare troppo. È ben noto che Federico, a seguito dell'incoronazione imperiale, fa formale domanda per essere accolto nella confraternita dell'ordine cistercense. Matteo Paris può così affermare:

"il più grande fra i principi della terra, colui che compì miracoli e trasformò il mondo, che venne assolto dalla scomunica che lo aveva colpito, dopo che, come si narra, aveva preso la veste di monaco cistercense ed aveva fatto penitenza in contrizione, umiliandosi ${ }^{\prime}{ }^{61}$

Tuttavia non si è del tutto certi che questa notizia possa essere vera, dato che la ricognizione del sepolcro non fornisce riscontri archeologici a conferma, ma testimonia l'uso delle insegne imperiali, seppur tradotte nel più modesto argento dorato. Elze stigmatizza quanto la vestizione dell'abito monastico possa essere ascritta piuttosto ad "uno schema letterario tipico", ${ }^{62}$ cosa che non può liquidare la condotta come fervida invenzione. Se il cronista riesce ad immaginare che un tale comportamento possa effettivamente rientrare nella complessa religiosità di Federico, nulla osta a darlo per buono o almeno per probabile.

Nondimeno la vestizione del saio deve essere considerata quale messaggio politico altamente meditato dal partito svevo: vuol significare la volontà del sovrano di riappacificarsi con la Chiesa in punto di morte. Federico si mostra così davvero ossequioso del sacerdozio, perché sa che entrambe le istituzioni, Chiesa ed Impero, sono espressioni strutturali del cosmo medievale e sono giustificate dalle elaborazioni culturali come effettivi prodotti della Provvidenza divina. Un'eventuale opzione che trova ragion d'essere nell'ordine soprannaturale. La morte sembra così obbligarlo ad una coerenza estrema verso gli etimi del suo ruolo. Nulla più di un tentativo volto all'affermazione della legittimità del proprio governo, ${ }^{63}$ quale espressione di "severo contegno che si era imposto come imperatore cristiano", stando a quanto ha già affermato Kantorowicz. ${ }^{64} \mathrm{La}$ scelta appare ancor più probabile se si considera che l'umiliazione in punto di morte va a rispondere ad un sentimento diffuso, che affida alla religione, anche a dispetto della gerarchia ecclesiastica, la mediazione del consenso. Pare così verosimile il ricorso a condotte sociali preventivamente codificate ed approvate.

Si comprende bene come questo messaggio politico sia volutamente orientato a ben predisporre il pubblico verso un sovrano che papa Innocenzo IV, suo nemico, addita come "babilonese", specie allorché giunge ad ordinare di cancellarne la dinastia. Ma come far collimare le due realtà, l'intento politico rivolto ad un élite e le adeguate

\footnotetext{
61 H.B. Luard (ed.), Mathei Parisiensis Cronica maiora, Rolls Series, Londra, 1876, p. 109.

62 Reinhard Elze, "La simbologia del potere nell'età di Federico II”, Federico II. Immagine e potere, Catalogo della Mostra, Bari, Castello Svevo, 4 febbraio-17 aprile 1995, M.S. Calò Mariani y R. Cassano (edd.), Marsilio, Venezia, 1995, pp. 45-51.

63 Salvatore Tramontana, Vestirsi e travestirsi..., p. 53.

64 Ernst Kantorowicz, I due corpi del sovrano, L'idea di regalità nella teologia politica medievale, Einaudi, Torino, 2012.
} 
strategie di rappresentazione verso un pubblico più vasto ed abituato a percepire una mise consona allo stato di potente?

Occorre necessariamente qualche correttivo, almeno rispetto ad un più ampio pubblico. Il popolo probabilmente non avrebbe accettato di vedere la salma del potente imperatore, dispensatore d'abbondanza, ora presentata ricoperta di umili stoffe, quasi fosse un monaco. Si può ragionevolmente sostenere che il Balio Manfredi, al fine di captare consenso e presentare il padre trapassato in una condizione adeguata all'officio rivestito in vita, abbia optato per un abbigliamento che rispecchia lo status imperiale, anche al costo di contravvenire alle disposizioni testamentarie paterne; ciò sempre se le si presuppongono veridiche. Difatti nel tragitto da Foggia a Palermo, con la sosta intermedia in Messina e la prothesis nella chiesa locale di Patti, si ravvisano più occasioni in cui la salma imperiale può essere abbigliata per essere adeguatamente presentata al pubblico. Durante una di queste si sarà ragionevolmente pensato di rivestirlo con abiti degni di un imperatore e con quella "veste di seta con disegni esotici" e il "mantello del mondo" che ha colpito l'immaginario degli astanti. E forse questi viene rivestito delle insegne proprie del rango già a Foggia, dove è ricomposto il cadavere ed è asportato il cuore per essere deposto nella Chiesa Matrice. Già in questa sede, con buona probabilità, non si è voluto generare lo spaesamento visivo nel popolo, che più volte ha potuto osservarlo vivo nello splendore delle cerimonie e soprattutto nelle opulente feste offerte durante i Parlamenti del Regno. Forse viene persino rivestito degli stessi abiti di cui si ha contegno attraverso Rosario di Gregorio. La vestizione attuata per la prothesis in Foggia appare essere l'opzione più ragionevole, giacché Spinelli informa, che nel transitare del feretro per Bitonto il sovrano è già coperto di un ricco velluto, che col suo rosso trionfale appare ben lontano dal bianco saio dei cistercensi. ${ }^{65}$

\section{FIG. 6 .}

\section{Salma di Federico II, xilografia da F. Daniele, I reali sepolcri di Palermo riconosciuti ed illustrati, Palermo, 1784, Tav. Q.}

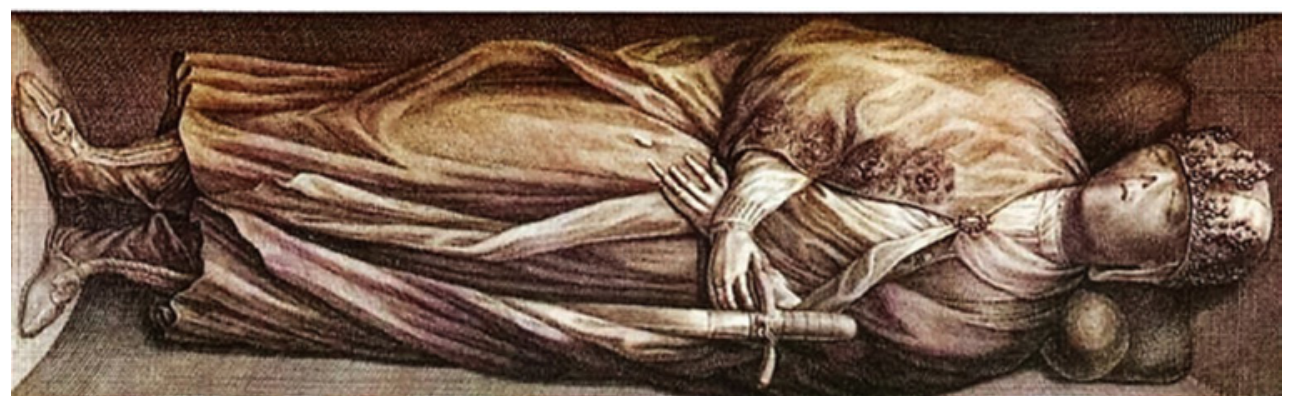

65 Emanuela Angiuli, "I vestiti dell'imperatore", Federico. Mito e memoria, E. Angiuli (ed.), Cittadella, Bari, 1994, pp. 129-145. 
FIG. 7.

Gallone con caratteri cufici posto a decoro dell'alba di Federico II, xilografia da F. Daniele, I reali sepolcri di Palermo riconosciuti ed illustrati, Palermo, 1784, Tav. R.

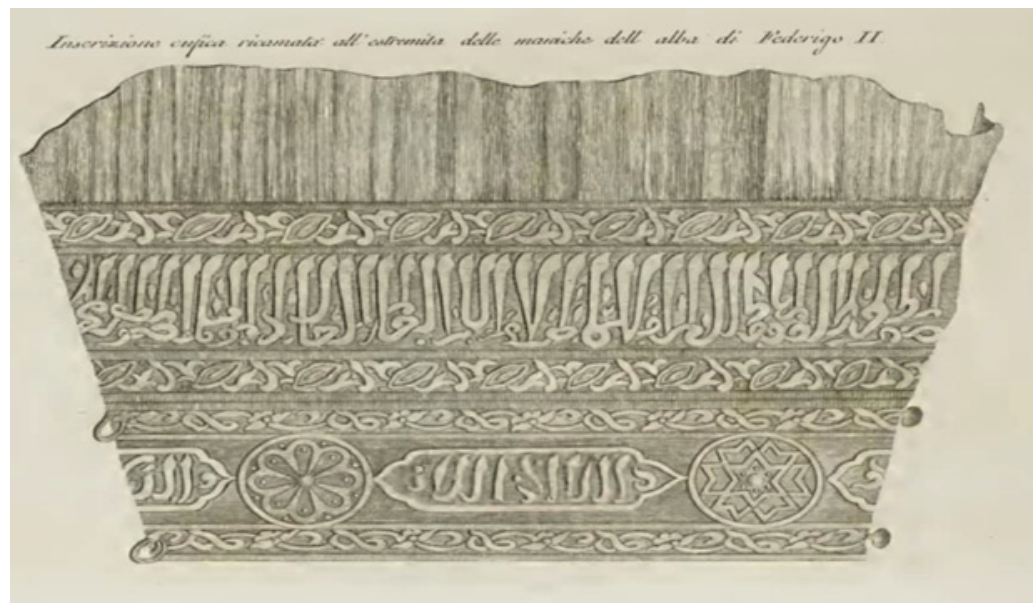

\section{La nudità come metafora della transitorietà del potere: un contrappeso materiale "retoricamente efficace"}

Tutto il valore dell'abbigliamento regio si ha a percepire ancora meglio, allorché si considerano le vicende che seguono la disfatta di Benevento e l'abbandono per ben tre giorni sul campo di battaglia del corpo di Manfredi, laddove giace privo delle insegne del rango. Al di là dei trucchi della narrazione storica fatti propri dal Villani per concludere con parecchia enfasi la tragedia umana dello scomunicato Manfredi e dell'intera famiglia sveva invisa alla Chiesa, appare opportuno riflettere sulla significatività dell'assenza delle insegne regie, nel controverso rito di espulsione dalla compagine sociale di questo sovrano. Un funerale che in ragione della scomunica si configura come un 'non' funerale, ma mero atto di pietà, più che altro dovuto, verso il cadavere. Un 'non' rito insomma, raffazzonato e dominato da una grande assenza: la mancanza delle insegne regali che devono contraddistinguere lo status di Manfredi. Per comprendere tutta la forza evocativa della loro carenza sul piano retorico e quanto questa assenza possa opporsi a quell'imaginerie connessa alla fenomenologia materiale della regalità, deve considerarsi lo stato delle elaborazioni della dottrina del potere sul tema del corpo nudo dei potenti a seguito della morte. Un tema proprio della riflessione della corte pontificia fin dalla metà del sec. XI, che ora fa sentire tutta la sua efficacia retorica anche nelle rappresentazioni della monarchia laica; cosa che ha implicazioni ben più profonde, perché investe quell'alea di legittimità a cui la veste stessa, come si è visto, viene preposta a garanzia. 
Si inaugura a mezzo del tema della nudità un discorso sulla caducità della monarchia, che stigmatizza l'eloquente assenza della veste e fa del corpo, privato dei segni del potere, l'espressione regina del sic transit gloria mundi. Un'assenza che costituisce un contrappeso materiale ai calmieri adoperati avverso la cesura dell'evento morte, espedienti fra cui la veste rientra. Una carenza che diviene un'espressione tangibile di quell'umiltà predicata dalla dottrina cristiana, quale soluzione riconducibile a quelle elaborazioni intellettuali che pongono degli argini agli eccessi dell'ambizione dei potenti; un calmiere a maggior ragione valido, allorché ci si rivolge ad un sovrano. La nudità di Manfredi sconfitto obbliga a far proprie le riflessioni fatte da Paravicini Bagliani sul corpo del papa denudato dei segni della potestà e, più in generale, della nudità come "metafora del potere" e delle sue alterne vicende, che contemplano nel termine ultimo imposto dalla morte la sua definitiva perdita. ${ }^{66}$

Deve osservarsi quanto fosse necessario all'economia della dottrina del potere del sec. XII un calmiere alla teorica onnipotenza dei sovrani, costituito da "contrappesi retoricamente efficaci", ${ }^{67}$ tali da rendere palese ad un pubblico erudito l'alto messaggio teoretico che la corte pontificia ha già fatto suo. Un discorso sulla caducità che fornisce soluzioni certamente confacenti alle categorie mentali del periodo, capaci di diventare subito loci autonomi, ancorché si inseriscono nei corredi retorici di quel "pallottoliere della regalità" a cui si suole ricorrere per offrire una descrizione più o meno incisiva della monarchia. Un tema certamente di successo dato il background condiviso, che viene incontro alle esigenze della nobiltas e del clero. Costoro sono capaci di gestire lo strumento della scrittura e vi possono ricorrere, allorché pongono in essere azioni di critica verso la monarchia o quando soltanto si limitano a fornire consigli sulla gestione del potere in opere come gli specula principum. Il tema costituisce per queste categorie uno strumento di garanzia, che può ampliare il loro raggio di azione rispetto allo strapotere esercitato dai re.

Occorre fare attenzione ad un altro dettaglio: l'espoliazione delle insegne, non a caso, è posta in essere nella cronaca di Villani da un uomo anonimo, di rango infimo, e come tale definito in maniera dispregiativa "ribaldo". Ciò ad enfatizzare quel discorso intorno alla caducità del potere, volto soprattutto a stigmatizzare l'impotenza a cui sono sottoposti persino i potenti del mondo a seguito della morte. Siamo così di fronte al più incisivo dei calmieri all'arbitrio regio, la possibilità che i sovrani vengano concessi al ludibrio della gente comune. Un avvertimento carico di paura, opposto al monarca quale opportuna 'clausola di salvaguardia' avverso gli abusi nell'esercizio del proprio officio. Un discorso sulla caducità che mette sotto i 'riflettori' una truce realtà a cui anche i re vanno incontro: con la morte il sovrano diviene uomo comune, perché da questo non ci si può aspettare più niente. Viene pure meno quel timore che obbliga agli atti di cortesia verso di lui. Privato di quell'espressione prima ideologica che materiale, capace di porlo

\footnotetext{
66 Agostino Paravicini Bagliani, Il corpo del Papa, Einaudi, Torino, 1994, pp. 89-91; Agostino Paravicini Bagliani, Le Chiavi e la Tiara... .

67 Ivi, 91.
} 
in uno 'spazio di copertura', anche un re può essere costretto a sopportare nel silenzio e nell'impotenza dello stato di salma le ingiustizie di altri uomini, per di più comuni. Anzi di quelli fra i più infimi sul piano sociale o ancor peggio su quello morale, come accade a Manfredi.

La nudità nei suoi termini più immediati, prima ancora d'essere metafora del potere diviene espressione materiale della condizione umana e delle miserie che l'accompagnano. Quale elaborazione retoricamente efficace vede nella vergogna d'essere nudo o dell'essere ridotti a tale stato, un contrappeso tanto ideologico quanto materiale, che si fa più incisivo se il denudato versa nello stato di scomunicato. Pertanto si può dire che la scomunica funge da 'catalizzatore' per l'attecchimento del tema entro l'opera di stigmatizzazione dei nemici della Chiesa.

La cronica del Villani con questo sentore può raccontare lo scempio del cadavere di Manfredi e la sua frettolosa sepoltura in terra sconsacrata in ragione della scomunica. Una sepoltura segnalata solo da un mero mucchio di sassi, poco adatto ai fasti di un re, ma assai efficace sul piano retorico per esplicitare quella caducità a cui i re non possono sottrarsi:

"Manfredi si cercò più di tre giorni, che non si ritrovava, e non si sapeva se fosse morto, o preso, o scampato, perché non aveva avuto a la battaglia indosso armi reali. Alla fine per uno ribaldo di sua gente fu riconosciuto per più insegne di sua persona in mezzo il campo ove fu la battaglia. E trovato il suo corpo per lo detto ribaldo, il mise traverso in su uno asino, venendo gridando: "Chi accatta Manfredi? Chi accatta Manfredi»; il quale ribaldo da uno barone del re fu battuto. E recato il corpo di Manfredi dinanzi al re, fece venire tutti i baroni ch'erano presi, e domandato ciascuno s'egli era Manfredi, tutti timorosamente dissero di si (....) molto ne fu commendato da' Franceschi, e per alquanti de' baroni del re fu pregato che gli facesse fare onore alla sepultura. Rispose il re: "Si feisse je volontiers, s'il non fust scomunié»; ma imperciò ch'era scomunicato, non volle il re Carlo che fosse recato in luogo sacro, ma appiè del ponte di Benevento fu seppellito, e sopra la sua fossa per ciascuno dell'oste gittata una pietra, onde si fece grande mora di sassi" ${ }^{68}$

Il locus della nudità, appena attecchito nella speculazione del potere statuale, trova efficacissimi esempi nella letteratura ecclesiastica, dove è richiamato in maniera martellante. Nonostante la nascita e lo sviluppo in un preciso contesto come la corte papale, la sua continua reiterazione non può essere del tutto estranea al pensiero laico tout court. Essa rientra sempre nei termini concernenti le ammonizioni tanto rituali, quanto consuete sugli ultimissimi, quali l'incombere della morte, che può giungere in un qualsiasi momento. Siamo così di fronte ad un problema retorico, che si muove entro le strutture del pensiero medievale.

68 Giovanni Villani, Nuova Cronica, 344, VII, 9. 
Una tale riflessione, sebbene considerata dal punto di vista della speculazione prodotta dai politologi laici, non può fare a meno di un riferimento preciso ad una predica del cardinale Odone di Châteauroux. Costui rivolgendosi al collegio cardinalizio svela uno degli arcani legati alla metafora della nudità e alla rituale espoliazione delle insegne a seguito della morte, quale rinuncia dovuta ad impedimento nello svolgere l'officio fino a quel momento ricoperto. In tal modo il cardinale avverte:

"noi saremo spogliati, prima di quanto pensiamo (...) di tutte le altre dignità di cui sono stati rivestiti i nostri predecessori, i quali poi ne sono stati spogliati (...). Preoccupiamoci dunque di non essere trovati nudi della veste delle virtù, della veste delle buone opere o della veste delle nozze, quando saremo spogliati delle dignità, onori e ricchezze, e della potestà, perché coloro che saranno trovati nudi di queste vesti non saranno ammessi alle nozze della gioia eterna" ${ }^{69}$

Di conseguenza la narrazione del Villani, mettendo in scena l'espoliazione del cadavere del proprio honor connesso allo status di re e stigmatizzando l'assenza delle regalia insigna, che rendono Manfredi riconoscibile per quel che è, offre piuttosto un mero ammaestramento morale, più che fornire un vero e proprio resoconto storico. La narrazione costituisce insomma il contraltare della predica del cardinale Odone di Châteauroux, anche perché si rivolge ad un più ampio pubblico. Si raffronta un trucco dello storico dunque, che non trova precedente più efficace che nell'alea dei gesti rituali adombrati dalla succitata predica, quali l'inequivoca espoliazione dei vestiti indicatori del rango proprio del morente o addirittura nello spogliarsi altrettanto rituale, come migliore locus d'umiltà, di colui che va incontro alla morte.

Il topos quale vera metafora della caducità dei potenti vuole esplicare non la mera nudità fisica, che nel testo del resto non appare mai apertis verbis, ma incide piuttosto la riflessione posta in essere dai politologi sui limiti intrinseci del potere. E se le insegne vogliono significare la funzione occupata in vita, non stupisce allora che Manfredi sconfitto in morte ne sia privato. Morte e sconfitta sono infatti equivalenti e rimandano entrambe all'abdicazione dal regno.

Le elaborazioni sul tema allora diventano più chiare attraverso un'altra evocazione retorica efficacissima concernente la metafora della nudità/espoliazione, che ritorna nella predica di Odone di Châteauroux:

"spogliato (...) dei suoi vestiti (...) Elzer figlio di Aaron, rivestì i vestiti (...) Elzer non dovette dubitare che sarebbe stato spogliato dei vestiti di cui era rivestito". ${ }^{70}$

69 David L. D’Avray y Jonathan Riley Smith y P. Cole, "Application of Theology to Current Affairs: Memorial Sermons on the Dead of Mansurah and on Innocent IV", Historical Research, 63 (1990), p. 239.

70 Ibidem. 
Si porta così in essere una sommessa dialettica fra valore della veste, significatività dei segni dello status ostentati in vita e gli effetti dell'evento morte sul potere detenuto. Pertanto il corredo dei segni diviene ancora più efficace durante la sua assenza e ciò accade proprio nel momento in cui lo si nega, sicché la sua essenzialità è fortemente ribadita sul piano retorico.

\section{Conclusioni}

Si può affermare che la veste costituisce una 'clausola di salvaguardia' materiale ed, al contempo, pone il defunto che la indossa in uno spazio di 'copertura sociale' connotato dal sistema di segni etero-referenziali di identità consoni al rango. Questa si inserisce pure in un sistema di produzione di senso che lascia poco spazio all'arbitrio destrutturante, ma si riassume nei limiti del socialmente approvato e condiviso. Il gusto per il dettaglio prezioso poi viene giustificato da una strategia di auto-rappresentazione portata in essere sin da Ruggero II, che tende alla spettacolarizzazione della monarchia locale. Pertanto l'uso di una veste preziosa viene sussunto in quel generale clima di corte che esige il dispendio di risorse da devolvere all'effimero. Una prodigalità che subisce una 'stretta' sotto Guglielmo I, che assume i caratteri del princeps clausus, ritorna con Guglielmo II e diventa, infine, 'marchio' dell'interfaccia fra Federico II imperatore e la compagine sociale. Una propensione alla profusione che viene negata al suo figlio naturale Manfredi, che perde col regno la vita. A questo rimane null'altro che la nudità del suo corpo, quale viva metafora del memento mori, allorché privato delle insegne del potere. Si apre ad una dialettica fra veste e nudità. Ciò contrappone l'abbigliamento, che costituisce una garanzia materiale rispetto alla legittimità dell'esercizio della monarchia, al corpo privato di questi segni e di ogni diritto sul regno. È dunque la nudità che svela un arcano delle strategie di auto-rappresentazione normanno-sveve: le vesti costituiscono la sublimazione visuale del rango ed assumono una significatività tutta funzionale all'equilibrio del sistema sociale elaborato dal Medioevo occidentale. Incarnano l'idea dell'officio ed hanno un valore non solo indicale, ma anche sostanziale, che tutto si conclude nella loro ostentazione. Tali segni rappresentano allora la falsificazione di un codice e si limitano a suggerire al fruitore una realtà idealizzata.

Questi segni ottimizzano altresì la trasmissione di informazioni ed obbligano il fruitore a quelle necessarie comportamentalità connesse al normale timor reverentiae del rango. Una tendenza che si rafforza di fronte all'evento morte. Allorquando dalla persona del sovrano defunto non ci si può più aspettare niente, sono proprio i segni d'eccellenza che hanno il compito di rimarcane la valenza dell'officio ricoperto e ne ribadiscono l'autorevolezza. La cura nella costruzione di un apparato di segni 'forti' ed adeguati al sovrano defunto deve necessariamente corrispondere ad un sentire diffuso. Questi svolgono una funzione performante rispetto alla percezione sociale, fomentano il gradimento della figura monarchica e si oppongono infine alla cesura dell'evento morte. 


\section{Bibliografia}

Abulafia, David, Federico II. Un imperatore medievale, Einaudi, Torino, 1993.

Andaloro, Maria (ed.), Nobiles Officinae: perle, filigrane e trame di seta dal Palazzo Reale di Palermo (Vol. 1-2), Maimone, Catania, 2006.

Andaloro, Maria, "Per la conoscenza e la conservazione delle tombe reali della Cattedrale di Palermo: linee storiche e storicoartistiche", Il sarcofago dell 'imperatore. Studi, ricerche e indagini sulla tomba di Federico II nella Cattedrale di Palermo 1994-1999, M. Andaloro (ed.), Regione Siciliana, Assessorato dei Beni Culturali, Ambientali e della Pubblica Istruzione, Palermo, 2002, pp. 135-148.

AndenNA, Giancarlo, "Dalla legittimazione alla sacralizzazione della conquista (10421140)", I caratteri originari della conquista normanna. Diversità e identità nel Mezzogiorno (1030-1130), Atti delle Sedicesime Giornate Normanno-Sveve, Bari, 5-8 ottobre 2004, Dedalo, Bari, 2006, pp. 371-405.

Angiul, Emanuela, "I vestiti dell'imperatore", Federico. Mito e memoria, E. Angiuli (ed.), Cittadella, Bari, 1994, pp. 129-145.

Arce, Javier, Funus imperatorum, Asgari, Madrid, 1989.

Arce, Javier, "Morte e apoteosi del principe. Imperatori divinizzati", Aurea Roma. Dalla città pagana alla città cristiana, S. Ensoli y E. La Rocca (edd.), L'Erma Di Bretschneider, Firenze, 2000, pp. 244-248.

BArone, Giulia, "La propaganda antiimperiale nell'Italia federiciana: l'azione degli Ordini Mendicanti”, Federico II e le città italiane, P. Toubert y A. Paravicini Bagliani (edd.), Sellerio, Palermo, 1994, pp. 278-289.

BAuer, Rotraud, "Il manto di Ruggero II", I Normanni popolo d'Europa. 1030-1200, Catalogo della Mostra, Roma, Palazzo Venezia, 28 gennaio-30 aprile 1994, M. D’Onofrio (ed.), Marsilio, Venezia, 1994, pp. 278-287.

BAuER, Rotraud, "Il manto di Ruggero II e le vesti regie", Nobiles Officinae: perle, filigrane e trame di seta dal Palazzo Reale di Palermo (Vol. 1-2), M. Andaloro (ed.), Maimone, Catania, 2006a, pp. 171-180.

BAUER, Rotraud, "Le vesti e le insegne per l'incoronazione dei re e degli imperatori del Sacro Romano Impero", Nobiles Officinae: perle, filigrane e trame di seta dal Palazzo Reale di Palermo (Vol. 1-2), M. Andaloro (ed.), Maimone, Catania, 2006b, pp. 425-429. Bourdieu, Pierre, La distinzione. Critica sociale del gusto, Il Mulino, Bologna, 1983.

Brandes, Wolfram, “Die 'Familie der Könige' in Mittelalter”, Diskussionsbeitrag zur Kritik eines vermeintlichen Erkenntnismodells, Rechtsgeschichte/Legal History, 21 (2013), pp. 262-284.

BRÜHL, Carlrichard, "Federico II: personalità di un sovrano", Federico II e il mondo Mediterraneo, P. Toubert y A. Paravicini Bagliani (edd.), Sellerio, Palermo, 1994, pp. 17-30. 
Calò Mariani, Maria S., "L'arte al servizio dello Stato", Federico II e il mondo Mediterraneo, P. Toubert y A. Paravicini Bagliani (edd.), Sellerio, Palermo, 1994, pp. 123-145. Calò Mariani, Maria S. y CASSANO, Raffaella (edd.), Federico II. Immagine e potere, Catalogo della Mostra, Bari, Castello Svevo, 4 febbraio-17 aprile 1995, Marsilio, Venezia, 1995.

Calò Mariani, Maria S., "I fenomeni artistici come espressione del potere", Potere, società e popolo tra età normanna ed età sveva (1189-1210), Atti delle Quinte Giornate Normanno-Sveve, Bari-Conversano, 26-28 ottobre 1981, Dedalo, Bari, 1983, pp. 215-250.

Calò Mariani, Maria S., "Immagine e potere", Federico II. Immagine e potere, Catalogo della Mostra, Bari, Castello Svevo, 4 febbraio-17 aprile 1995, M. S. Calò Mariani y R. Cassano (edd.), Marsilio, Venezia, 1995, pp. 39-43.

Cammarosano, Paolo, "Immagine visiva e propaganda nel Medioevo", I linguaggi della propaganda. Studio di casi: Medioevo, Rivoluzione Inglese, Italia liberale, Fascismo, Resistenza, Mondadori, Milano, 1991, pp. 8-29.

Cantarella, Glauco Maria, La Sicilia e i Normanni. Le fonti del mito, Patron, Bologna, 1988.

Cantarella, Glauco Maria, Principi e corti. L'Europa del XII secolo, Einaudi, Torino, 1997.

Cantarella, Glauco Maria, "Historia non facit saltus? Gli imprevisti normanni", I re nudi. Congiure, assassini, tracolli ed altri imprevisti nella storia del potere, G. M. Cantarella y F. Santi (edd.), Centro Italiano di Studi sull'Alto Medioevo, Spoleto, 1996, pp. 9-38.

Cantarella, Glauco Maria, Medioevo. Un filo di parole, Mondadori, Milano, 2002.

Cantarella, Glauco Maria, "Le basi concettuali del potere", Per me reges regnant. La regalità sacra nell'Europa medievale, F. Cardini y M. Saltarelli (edd.), il CerchioCantagalli, Rimini-Siena, 2002, pp. 193-207.

Cantarella, Glauco Maria, “Divagazioni preliminari”, «C'era una volta un re...» Aspetti e momenti della regalità, Seminario del Dottorato in Storia Medievale dell'Università di Bologna, Bologna, 17-18 dicembre 2003, G. Isabella (ed.), Clueb, Bologna, 2005, pp. 9-24.

Cantarella, Glauco Maria, "Il pallottoliere della regalità: il perfetto re della Sicilia normanna", Studi in onore di Vincenzo D'Alessandro, Dentro e fuori la Sicilia, P. Corrao y I. E. Mineo (edd.), Viella, Roma, 2009, pp. 29-44.

Carile, Rocco Antonio, Produzione e usi della porpora nell'impero bizantino, Istituto Veneto di Scienze, Lettere ed Arti, Venezia, 1998.

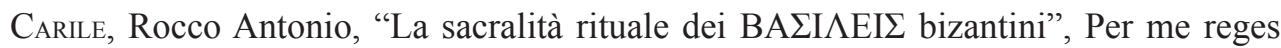
regnant. La regalità sacra nell'Europa medievale, F. Cardini y M. Saltarelli (edd.), il Cerchio-Cantagalli, Rimini-Siena, 2002, pp. 53-95. 
CARdini, Franco, "Federico II. La memoria e il mito", Federico II. Immagine e potere, Catalogo della Mostra, Bari, Castello Svevo, 4 febbraio-17 aprile 1995, M. S. Calò Mariani y R. Cassano (edd.), Marsilio, Venezia, 1995, pp. 453-454.

Cilento, Adele y Burgarella, Filippo, Bisanzio in Sicilia e nel sud dell'Italia, Magnus, Udine, 2005.

Delle Donne, Fulvio, Il potere e la sua legittimazione: letteratura encomiastica in onore di Federico II di Svevia, Nuovi Segnali, Arce, 2005.

Delle Donne, Fulvio, "Liturgie del potere. Le testimonianze letterarie", Nascita di un regno. Poteri signorili, istituzioni feudali e strutture sociali nel Mezzogiorno normanno (1130-1194), Atti delle diciassettesime Giornate normanno-sveve, Bari, 10-13 ottobre 2006, R. Licinio y F. Violante (edd.), Dedalo, Bari, 2008, pp. 331-368.

Delogu, Paolo, "Idee sulla regalità: l'eredità normanna", Potere, società e popolo tra età normanna ed età sveva (1189-1210), Atti delle Quinte Giornate Normanno-Sveve, Bari-Conversano, 26-28 ottobre 1981, Dedalo, Bari, 1983, pp. 185-214.

Delogu, Paolo, "La committenza degli Altavilla: produzione monumentale e propaganda politica", The Mosaics of Norman Sicily, O. Demus (ed.), Hacker Art Books, New York, 1988.

Didi-Huberman, Georgess, "Imitation, représentation, fonction. Remarques sur un mythe épistémologique”, L’image. Fonctions et usage des images dans l'Occident médiéval, Actes du 6e International Workshop on Medieval Societes, Erice, 17-23 octobre 1992, J. Baschet y J.C. Schmitt (edd.), Le Léopard d'Or, Paris, 1996, pp. 59-86.

Dölger, Franz, "Die Familie der Könige im Mittelalter", Historisches Jahrbuch 60 (1940), pp. 397-420.

DölgER, Franz, "Brüderlichkeit der Fürsten", Reallexikon für Antike und Christentum, vol. 2, Hiersemann, Stuttgart, 1954, col. 642.

D'onofrio, Mario (ed.), I Normanni popolo d'Europa. 1030-1200, Catalogo della Mostra, Roma, Palazzo Venezia, 28 gennaio-30 aprile 1994, Marsilio, Venezia, 1994, pp. 188-192.

Elze, Reinhard, "Insegne del potere sovrano e delegato in Occidente", Simboli e simbologia nell'Alto Medioevo, Atti della XXIII Settimana di Studio del Centro Italiano di Studi sull'Alto Medioevo, Spoleto, 3-9 aprile 1975, Centro Italiano di Studi sull'Alto Medioevo, Spoleto, 1976, pp. 569-593.

Elze, Reinhard, "Tre Ordines per l'incoronazione di un re e di una regina del regno normanno di Sicilia", Atti del Congresso Internazionale di Studi sulla Sicilia Normanna, Palermo, 4-8 dicembre 1972, Istituto di Storia Medievale Università di Palermo, Caltanissetta-Roma, Sciascia, 1973, pp. 438-459.

Elze, Reinhard, “La simbologia del potere nell'età di Federico II”, Federico II. Immagine e potere, Catalogo della Mostra, Bari, Castello Svevo, 4 febbraio-17 aprile 1995, M.S. Calò Mariani y R. Cassano (edd.), Marsilio, Venezia, 1995a, pp. 45-51. 
Elze, Reinhard, "Le insegne del potere", Strumenti, tempi e luoghi di comunicazione nel Mezzogiorno normanno-svevo, Atti delle Undicesime Giornate Normanno-Sveve, Bari, 26-29 ottobre 1993, Dedalo, Bari, 1995b, pp. 113-129.

Grabar, André, L'empereur dans l'art byzantin. Recherches sur l'art officiel de l'Empire d'Orient, Les Belles Lettres, Paris, 1936.

Herklotz, Ingo, "Lo spazio della morte e lo spazio della sovranità", I Normanni popolo d'Europa. 1030-1200, Catalogo della Mostra, Roma, Palazzo Venezia, 28 gennaio-30 aprile 1994, M. D’Onofrio (ed.), Marsilio, Venezia, 1994, pp. 320-326.

Houben, Hubert, Ruggero II di Sicilia. Un sovrano tra Oriente e Occidente, La Terza, Roma- Bari, 1999.

D’avray, David L. y Riley Smith, Jonathan y Cole, P., “Application of Theology to Current Affairs: Memorial Sermons on the Dead of Mansurah and on Innocent IV", Historical Research, 63 (1990), pp. 227-239

Iorio, Raffaele, "Umanità disumana di Federico persona e personaggio", Federico II. Mezzogiorno, Atti del Convegno Internazionale di Studio, Potenza-Avigliano-Castel Lagopesole- Melfi, 18-23 ottobre 1994, C.D. Fonseca (ed.), Edizioni de Luca, Roma, 1999, pp. 701-748.

Johns, Jeremy, "Le iscrizioni e le epigrafi in arabo", Nobiles Officinae: perle, filigrane e trame di seta dal Palazzo Reale di Palermo (Vol. 1-2), M. Andaloro (ed.), Maimone, Catania, 2006, pp. 119-131.

Kantorowicz, Ernst, "On the Portrait of Roger II in the Martorana in Palermo", Proporzioni. Studi di storia dell'arte, III, Sansoni, Firenze, 1950, pp. 30-35.

Kantorowicz, Ernst, I mosaici di Monreale, Flaccovio, Palermo, 1960.

Kantorowicz, Ernst, "Some Reflections on Portraiture in Byzantine Art", The Art of Byzantium and the Medieval West: Selected Studies", W.E. Kleinbauer (ed.), Indiana University Press, Bloomington-London, 1976, pp. 256-269.

Kantorowicz, Ernst, "The Byzantine Contribution to Western Art of the Twelfth and Thirteenth Centuries", The Art of Byzantium and the Medieval West: Selected Studies, W.E. Kleinbauer (ed.), Indiana University Press, Bloomington-London, 1976, pp. 357-388.

Kantorowicz, Ernst, I mosaici di Santa Maria dell'Ammiraglio a Palermo, Istituto Siciliano di Studi Bizantini e Neoellenici, Palermo, 1990.

Kantorowicz, Ernst, "La Cappella Palatina di Palermo. I mosaici del Presbiterio", I mosaici del periodo normanno in Sicilia, E. Kitzinger (ed.), Istituto Siciliano di Studi Bizantini e Neoellenici, Palermo, 2000a.

Kantorowicz, Ernst, "La cattedrale di Cefalù. La cattedrale di Palermo e il Museo Diocesano. Mosaici profani”, I mosaici del periodo normanno in Sicilia, VI, E. Kitzinger (ed.), Istituto Siciliano di Studi Bizantini e Neoellenici, Palermo, 2000b.

Kantorowicz, Ernst, Laudes Regiae. Uno studio sulle acclamazioni liturgiche e sul culto del sovrano nel Medioevo, Medusa, Milano, 2006. 
Kantorowicz, Ernst, I due corpi del sovrano, L'idea di regalità nella teologia politica medievale, Einaudi, Torino, 2012.

KöNIG, René, Il potere della moda, Liguori, Napoli, 1992.

LIPINSKY, Angelo, "Le insegne regali dei sovrani di Sicilia e la scuola orafa palermitana", Atti del Congresso Internazionale di Studi sulla Sicilia Normanna, Palermo, 4-8 dicembre 1972, Caltanissetta-Roma, Istituto di Storia Medievale Università di Palermo, Sciascia, 1973, pp. 162-194.

Lipinsky, Angelo, Oro, argento, gemme e smalti. Tecnologia delle arti dalle origini alla fine del Medioevo. 3000 a. C. - 1550 d. C., Olschki, Firenze, 1975.

MacCormack, Sabine G. (tr. It.), Arte e cerimoniale nell'antichità, Einaudi, Torino, 1995.

MÉNAGER, Léon-Robert, "L'institution monarchique dans les États normands d'Italie. Contribution à l'étude du pouvoir royal dans les principautés occidentales, aux XI-XII siècles", Hommes et institutions de l'Italie normande, Léon-Robert Ménager (ed.), London, 1981, pp. 303-331, 445-468.

Mertens, Dieter, Il pensiero politico medievale, Il Mulino, Bologna, 1999.

Paravicini Bagliani, Agostino, Il corpo del Papa, Einaudi, Torino, 1994.

Paravicini Bagliani, Agostino, Le Chiavi e la Tiara. Immagini e simboli del papato medievale, Viella, Roma, 1998.

Pertusi, Agostino, "Insegne del potere sovrano e delegato a Bisanzio e nei paesi di influenza bizantina", Simboli e simbologia nell'Alto Medioevo, Atti della XXIII Settimana di Studio del Centro Italiano di Studi sull'Alto Medioevo, Spoleto, 3-9 aprile 1975, Centro Italiano di Studi sull'Alto Medioevo, Spoleto, 1976, pp. 481-568.

Price, Simon R. F., Rituals and Power. The Roman Imperial Cult in AsiaMinor, Cambridge, 1994.

Schneider, Jane, "The Antropology of Cloth", Annual Review of Anthropology, 16 (1987), pp. 409-448.

Schramm, Percy Ernst, "Herrschaftszeichen und Staatssymbolik. Beiträge zu ihrer Geschichte von dritten bis zum sechzehnten Jh., I-III", Early Medieval Europe, Vol. 3, Stuttgart, 1956, pp. 135-156.

Schramm, Percy Ernst, Kaiser Friedrichs II. Herrschaftszeichen, Vandenhoeck \& Ruprecht, Goettingen, 1955.

Simmel, Georg, Metropoli e moda, Piano B., Prato, 2011.

Tabacco, Giovanni, Le ideologie politiche del Medioevo, Einaudi, Torino, 2000.

Tramontana, Salvatore, L'effimero nella Sicilia normanna, Sallerio, Palermo, 1984.

Tramontana, Salvatore, Vestirsi e travestirsi in Sicilia. Abbigliamento, feste e spettacoli nel Medioevo, Sellerio, Palermo, 1993. 
Tramontana, Salvatore, "Comunicare nel Mezzogiorno", Strumenti, tempi e luoghi di comunicazione nel Mezzogiorno normanno-svevo, Atti delle Undicesime Giornate Normanno-Sveve, Bari, 26-29 ottobre 1993, Dedalo, Bari, 1995, pp. 9-30.

TRAmontana, Salvatore, "Popoli, etnie e mentalità alla vigilia della conquista di Sicilia", I caratteri originari della conquista normanna. Diversità e identità nel Mezzogiorno (1030-1130), Atti delle Sedicesime Giornate Normanno-Sveve, Bari, 5-8 ottobre 2004, Dedalo, Bari, 2006, pp. 86-107.

Tronzo, William, The Cultures of His Kingdom. Roger II and the Cappella Palatina in Palermo, Princeton University Press, Princeton, 1997. Vagnoni, Mirko, Federico II allo specchio. Analisi iconografica e politico funzionale delle sue raffigurazioni, Tesi di Laurea in Storia, Università degli Studi di Siena, Anno Accademico 2003-2004.

VAGNONI, Mirko, "Il significato politico delle caratteristiche iconografiche di Federico II di Svevia", Iconographica. Rivista di iconografia medievale e moderna 5 (2006), pp. 64-75.

VAGnonI, Mirko, "La legittimità e la sacralità imperiale di Federico II di Svevia", Tabulae. Centro Studi Federiciani 18 (2006), pp. 127-169.

VAGNoni, Mirko, "Problemi di legittimazione regia: 'imitatio Byzantii'", Il papato e $i$ Normanni: temporale e spirituale, convegno di studi, Ariano Irpino 6-7 dicembre 2007, E. D'Angelo y C. Leonardi (edd.), SISMEL edizioni del Galluzzo, Firenze, 2011, pp. 50-65.

VAGnoni, Mirko, Le rappresentazioni del potere. La sacralità dei normanni di Sicilia un mito?, Edizioni Caratteri Mobili, Bari, 2012.

VAGnoni, Mirko, Dei gratia rex Sicilie. Scene d'incoronazione divina nell'iconografia regia normanna, Federico II University Press, Napoli, 2017.

Varoli Piazza, Rosalia, "La produzione di manufatti tessili nel Palazzo Reale di Palermo: 'tiraz' o 'ergasterion'”, I Normanni popolo d'Europa. 1030-1200, Catalogo della Mostra, Roma, Palazzo Venezia, 28 gennaio-30 aprile 1994, M. D’Onofrio (ed.), Marsilio, Venezia, 1995, pp. 288-290.

Varoli Piazza, Rosalia, "Per un approccio interdisciplinare ai manufatti tessili antichi", Nobiles Officinae: perle, filigrane e trame di seta dal Palazzo Reale di Palermo (Vol. 1-2), M. Adaloro (ed.), Maiomone, Catania, 2006, pp. 393-395. 
